\title{
Common Rules Guide Comparisons of Speed and Direction of Motion in the Dorsolateral Prefrontal Cortex
}

\author{
Cory R. Hussar ${ }^{1}$ and Tatiana Pasternak ${ }^{1}$ \\ ${ }^{1}$ Department of Neurobiology and Anatomy, University of Rochester, Rochester, New York 14642
}

When a monkey needs to decide whether motion direction of one stimulus is the same or different as that of another held in working memory, neurons in dorsolateral prefrontal cortex (DLPFC) faithfully represent the motion directions being evaluated and contribute to their comparison. Here, we examined whether DLPFC neurons are more generally involved in other types of sensory comparisons. Such involvement would support the existence of generalized sensory comparison mechanisms within DLPFC, shedding light on top-down influences this region is likely to provide to the upstream sensory neurons during comparison tasks. We recorded activity of individual neurons in the DLPFC while monkeys performed a memory-guided decision task in which the important dimension was the speed of two sequentially presented moving random-dot stimuli. We found that many neurons, both narrow-spiking putative local interneurons and broad-spiking putative pyramidal output cells, were speed-selective, with tuning reminiscent of that observed in the motion processing middle temporal (MT) cortical area. Throughout the delay, broad-spiking neurons were more active, showing anticipatory rate modulation and transient periods of speed selectivity. During the comparison stimulus, responses of both cell types were modulated by the speed of the first stimulus, and their activity was highly predictive of the animals' behavioral report. These results are similar to those found for comparisons of motion direction, suggesting the existence of generalized neural mechanisms in the DLPFC subserving the comparison of sensory signals.

\section{Introduction}

Many studies of the neural basis of perceptual decisions have used relatively simple sensory-motor tasks, where subjects made eye movements to report their decision about stimulus identity. These studies manipulated detectability of these stimuli while recording from neurons in a number of extrastriate cortical areas as well as from parietal and prefrontal regions, revealing a reliable link between neuronal activity and behavioral report (Gold and Shadlen, 2007; Nienborg et al., 2012). Another form of perceptual decision-making, perhaps even more ubiquitous, is when subjects make their choices based on the comparison between the current stimulus and the stimulus held in working memory. We have been examining neuronal activity during memory-guided comparisons by recording from the dorsolateral prefrontal cortex (DLPFC) of monkeys comparing directions of two sequentially presented random-dot moving stimuli. We focused on DLPFC because it has been strongly implicated in active maintenance of task relevant signals and is thought to give rise to the top-down influences to the upstream sensory neurons, an impor-

\footnotetext{
Received Aug. 24, 2012; revised Nov. 26, 2012; accepted Nov. 27, 2012.

Author contributions: T.P. designed research; C.R.H. and T.P. performed research; C.R.H. analyzed data; C.R.H. and T.P. wrote the paper.

This work was supported by Grant R01 EY11749 from the National Eye Institute and Grant P30 EY01319 from the Center for Visual Science. We thank Marc Mancarella for technical assistance, Martin Gira for help with electronics, Leo Lui for help with data collection, and James Bisley, Ben Hayden, and Bart Krekelberg for comments and useful suggestions on the manuscript.

The authors declare no competing financial interests.

Correspondence should be addressed to Dr. Tatiana Pasternak, Department of Neurobiology \& Anatomy, Box 603, University of Rochester, Rochester, NY 14642. E-mail: tania@cvs.rochester.edu.

DOI:10.1523/JNEUROSCI.4075-12.2013

Copyright $\odot 2013$ the authors $\quad 0270-6474 / 13 / 330972-15 \$ 15.00 / 0$
}

tant aspect of its role in executive control (Miller and Cohen, 2001). We found that this region, directly interconnected with the motion processing area MT (Barbas, 1988; Schall et al., 1995; Petrides and Pandya, 2006), actively participates in all components of the direction comparison task (Zaksas and Pasternak, 2006; Hussar and Pasternak, 2009, 2012). Specifically, many neurons showed direction selective (DS) responses to visual motion, carried reliable but transient delay activity reflecting the remembered direction, and their responses during the comparison stimulus were modulated by that direction. Importantly, the activity of neurons showing such comparison effects (CEs) was predictive of upcoming perceptual decisions, for the first time, establishing a link between comparison signals and perceptual reports.

Visual motion of an object is defined not only by its direction but also by its speed, and these two attributes are closely linked in both their sensory and neuronal representations (Maunsell and Van Essen, 1983; Mikami et al., 1986b; Pasternak, 1987), most likely arriving in the DLPFC via the same pathways. Having characterized participation of DLPFC in direction comparisons, we examined whether the rules that guide the representation of stimulus speed during speed comparisons are similar. We found that during the speed task the patterns of activity paralleled those observed during the direction task. Many neurons, both narrowspiking (NS) putative interneurons and broad-spiking (BS) putative pyramidal cells, were speed-selective and their tuning resembled tuning recorded in MT. Throughout the delay, periods of selectivity were transient, and in late delay this selectivity was more prominent in putative pyramidal cells. During the second stimulus, responses of many neurons showed CEs that decreased with the difference between the two stimuli, and their activity was 


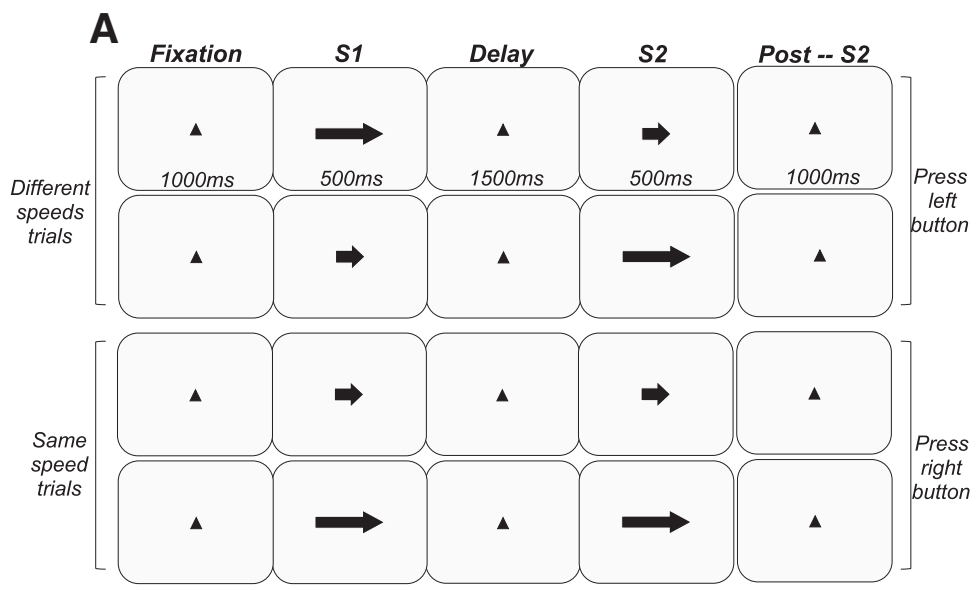

B

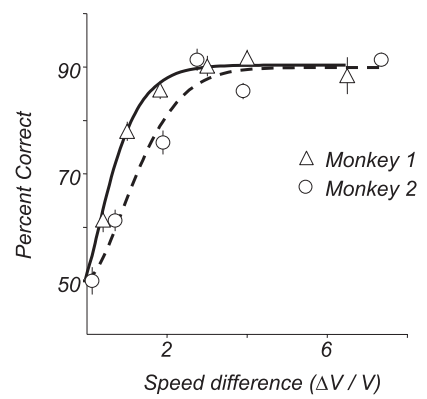

C

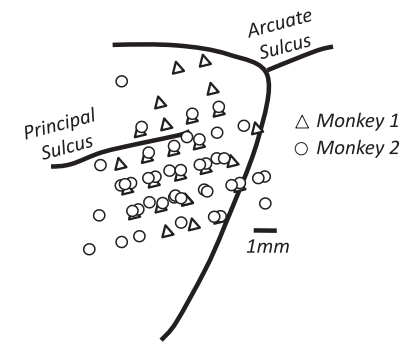

Figure 1. Behavioral task; performance; recording sites. A, Speed discrimination task. Animals reported whether coherently moving random-dot stimuli presented during $\mathrm{S} 1$ and $\mathrm{S} 2$ stimuli moved at the same or different speeds by pressing one of the two response buttons. On each trial, a stimulus moving at base speed $(2$ or $4 \% s)$, indicated by shorter arrows, was compared with a stimulus moving either at the same speed (two bottom rows) or at a higher speed (two top rows). The base and the faster speeds could appear at random either during S1 or S2. On trials with S1 and S2 moving at the same speed, stimuli moved either at base speeds (third row) or at any of the comparison speeds (bottom row). These two types of trials (same and different) were randomly interleaved, and comparison speeds were chosen to bracket the animal's threshold. $\boldsymbol{B}$, Average psychometric functions for the two monkeys, based on 30,142 trials collected during 150 recording sessions. The average performance of each animal is plotted as a function of speed differences between $S 1$ and $S 2$, expressed as a Weber fraction $(\Delta V / V)$, where $\Delta V$ is speed increment and $V$ is base speed. C, Locations of all task-related neurons recorded in each animal indicated by triangles (Monkey 1 ) and circles (Monkey 2). Recordings were clustered between the principal and the arcuate sulcus, with the larger proportion of neurons located in the ventral region.

predictive of the animal's choice. These results provide the first characterization of both sensory and memory-related speedselective activity in the DLPFC, illustrating the precision with which its neurons represent this fundamental dimension of visual motion. They also established a relationship between the CEs and perceptual reports analogous to that observed during the direction comparison task. This similarity in neuronal activity during comparisons of speeds and directions points to a common mechanism underlying perceptual decisions across various sensory dimensions.

\section{Materials and Methods}

Subjects

We recorded neuronal activity in the dorsolateral PFC of two adult male macaque monkeys. All training, surgery, and experimental procedures were performed in accordance with the National Institutes of Health Guide for the Care and Use of Laboratory Animals and were approved by the University of Rochester Committee for Animal Research. Animals received their water during times of behavioral testing each weekday, and food was always available in their home cages. Body weights were measured and recorded on a daily basis to monitor health and growth of the animals.

\section{Visual stimuli and eye position}

Stimuli were presented on a video monitor (19-inch IIyama Vision Master Pro-513, running at $1152 \times 870$-pixel resolution and a 75 $\mathrm{Hz}$ refresh rate) placed $57 \mathrm{~cm}$ in front of the monkeys. They consisted of random dots placed in a circular $4^{\circ}$ aperture with the density set at $4.7 \mathrm{dots} / \mathrm{deg}^{2}$. The dots were $0.03^{\circ}$ of visual angle in diameter with a luminance of 15 $\mathrm{cd} / \mathrm{m}^{2}$, shown on a dark background of $0.1 \mathrm{~cd} /$ $\mathrm{m}^{2}$. Each dot persisted for the entire duration of the stimulus. All stimuli were presented at the fovea, at the center of the display superimposed on the fixation point.

For all experiments, eye position was monitored with an infrared video eye-tracking package (ISCAN). Daily calibration was performed before each recording session, requiring animals to briefly fixate for a variable amount of time (100-1000 ms) while the offsets and gains were adjusted. Throughout all subsequent behavioral sessions, animals were required to maintain fixation within $2^{\circ}$ of a centrally presented fixation dot. Any trials in which this condition was not met were immediately aborted and animals were required to reinitiate a trial. Data from these aborted trials were discarded.

\section{Behavioral tasks}

During each recording session, animals were presented with a battery of behavioral tasks. The animals performed a coarse direction discrimination task $\left(90^{\circ}\right.$ differences in direction) while we searched for recordable neurons. Once a unit was isolated, its tuning for direction was evaluated in a block of 40 trials of this task (for details, see Cell selection, below). After direction selectivity was established, two directions, preferred and antipreferred, were chosen to be used in the two accuracy tasks, direction, and speed discriminations, each run in separate blocks of $\sim 200$ trials each. These two tasks were followed by a $\sim 200$ trial passive fixation task. In most cases, the direction task preceded the speed task and the passive fixation task was usually run last. In this report, we are focusing on the activity of DLPFC neurons during the speed discrimination and passive fixation tasks. Neuronal activity recorded during the direction discrimination task can be found in previous reports (Hussar and Pasternak, 2009; Hussar and Pasternak, 2010; Hussar and Pasternak, 2012).

Speed discrimination task. The structure of this task is outlined in Figure $1 \mathrm{~A}$. Each trial began with the presentation of a small triangle fixation cue. Animals initiated each trial by holding fixation within a small window $\left( \pm 1.5^{\circ}\right)$ for a $1000 \mathrm{~ms}$. After this, they were required to maintain fixation throughout the periods of S1, delay, S2, and postS2. After post-S2, the fixation dot was extinguished and animals reported whether the speeds of the two stimuli, S1 and S2, were the same or different by pressing one of the two response buttons. Task difficulty was controlled by varying differences in speed between S1 and S2 (Fig. 1B). Discriminations were made between a base speed $(2$ or $4 \%$ s) and a comparison speed (ranging from 2.5-16\%). Comparison speeds were either greater or the same as the base speed and their exact values were chosen to bracket the animal's threshold. The base speed used in each recording session was matched to that used during the direction task. On each trial, S1 and S2 moved in the same direction, but between trials the directions were chosen at random between two directions previously identified as preferred and antipreferred. 
Passive fixation task. During this task, the stimuli and the time of their appearance were identical to the speed discrimination task, with S1 and S2 stimulus combinations matching the preceding speed discrimination task (see Fig. 5A). Animals were cued with a small " $x$ " fixation point and were rewarded for maintaining fixation throughout the trial. No button response was required.

\section{Physiological recordings}

Recording locations (Fig. 1C) were identified from structural MRI scans. Data from a subset of these locations have been previously analyzed and reported (Hussar and Pasternak, 2009; Hussar and Pasternak, 2010; Hussar and Pasternak, 2012). All recordings were performed using a single tungsten glass-coated microelectrode (0.5-3 M $\Omega$; Alpha Omega Engineering). Electrodes were positioned over the chamber-enclosed craniotomy using a cilux grid with 1-mm-spaced openings (Crist Instruments). Custom-made steel guide tubes were inserted into the hole chosen for each day's recording to provide structural stability. Guide tubes were lowered to the dura but did not penetrate. Electrodes were driven through the dura using either a hydraulic micromanipulator (Narashigi Group) or a NAN electrode drive (NAN Instruments).

Cell selection. Cells were selected while the monkey performed a direction discrimination task during which the directions of S1 and S2 were either $90^{\circ}$ apart or the same. The durations of the two stimuli and the length of the delay were identical to other tasks, $500 \mathrm{~ms}$ and $1500 \mathrm{~ms}$, respectively. During this task, eight equally spaced motion directions were used. Single units were selected for further recording if they were clearly differentiated from background noise. Once isolated, the neurons were formally evaluated for task-related activity, defined as significant deviation of firing rates at any point in the trial from baseline activity (Wilcoxon sign-rank test, $p<0.01$ ). As baseline we chose activity during a $200 \mathrm{~ms}$ bin of fixation, centered at $500 \mathrm{~ms}$ leading to trial initiation. This was necessary because many PFC neurons change their firing rates immediately before $\mathrm{S} 1$ onset.

Cell classification. Previous intracellular recordings identified a relationship between certain metrics of a neuron's extracellularly recorded action potential and underlying morphology (McCormick et al., 1985; Contreras and Palmer, 2003). They showed that action potentials of pyramidal neurons have longer durations than interneurons. Subsequently, a number of extracellular recording studies used waveform durations to classify neurons in the $\mathrm{PFC}$ and in other cortical regions into putative pyramidal neurons and putative interneurons (Mitchell et al., 2007; Diester and Nieder, 2008; Johnston et al., 2009). In a prior study, we used waveform durations to classify cells as putative interneurons and pyramidal cells (Hussar and Pasternak, 2009). In that paper, we provided a detailed account of the methods used for classifying the two cell types and showed that the distribution of action potential durations for all recorded neurons was significantly nonunimodal (Hartigan's dip test, $p=0.02$ ) (Hartigan and Hartigan, 1985; Hussar and Pasternak, 2009, their Figure $1 \mathrm{H})$. Because the current analysis is based on recordings from these same neurons, here we provide only a brief summary of this classification. Waveforms were isolated and saved with Plexon sorting software. For each neuron, an average waveform was derived and interpolated with a spline fit to a precision of $2.5 \mu$ s (Mitchell et al., 2007), and waveform duration was determined by measuring the time between the trough and the peak. The category boundary was based on the trough of the bimodal distribution, at $200 \mu \mathrm{s}$. This value was used to divide the cells into two classes: neurons with durations $<200 \mu$ s were classified as NS putative inhibitory interneurons and neurons with waveform durations $>200 \mu$ s were grouped as BS putative pyramidal neurons. Additional care was taken to avoid cell misclassifications (for further details see Hussar and Pasternak, 2009).

\section{Data analysis}

Responses during S1. All neurons were first evaluated for stimulus-related responses, defined as any significant deviation of firing rates from baseline activity (Wilcoxon sign-rank test $p<0.01$ ) during the stimulus presentation. For this analysis, baseline activity was defined as the average activity in a $200 \mathrm{~ms}$ bin immediately before stimulus onset. For all neurons with significant stimulus responses, activity for each of the pre- sented speeds was evaluated independently. The speed that produced the greatest deviation from baseline was termed the "most-preferred" speed, whereas the speed resulting in the smallest deviation from baseline was labeled the "least-preferred" speed. The difference between responses elicited by these two speeds was quantified with ROC analysis (see Speed selectivity, below).

Direction selectivity. Selectivity for direction was evaluated with ROC analysis by comparing responses to the preferred and antipreferred directions recorded during the direction discrimination task. This analysis provided area below the ROC curve (AROC) values, ranging between 0 and $1 . \mathrm{AROC}=0.5$ indicated that activity associated with each of the two directions was indistinguishable, whereas AROC $>0.5$ indicated higher activity during trials containing preferred direction. Conversely, AROC $<0.5$ indicated higher activity on antipreferred trials. Significance of AROCs was evaluated by bootstrap test, shuffling preferred and antipreferred trial labels (Efron and Tibshirani, 1993). This process was repeated 1000 times, generating a distribution of shuffled AROC values. The original AROC value was deemed significant if it fell within the top or bottom $2.5 \%$ of the shuffled distribution $(p<0.05)$. Categorization of neurons into DS and non-DS groups was evaluated with a sliding significance test. Significance was evaluated in a $100 \mathrm{~ms}$ window stepped in 100 $\mathrm{ms}$ intervals throughout $\mathrm{S} 1$. If any window within this time period (50$550 \mathrm{~ms}$ ) was found to be significant, a neuron was classified as DS.

Speed selectivity. Selectivity for speed was quantified by computing AROCs generated by comparing firing rates on trials with "mostpreferred" and "least-preferred" speeds presented during S1 and/or S2. It was calculated by sliding a $100 \mathrm{~ms}$ window in $100 \mathrm{~ms}$ increments along the spike train. During the speed task, all speeds were presented in both the preferred and antipreferred direction. All activity was scored to the mean and SD of the distribution of responses to the preferred and antipreferred directions. All AROC values for speed selectivity were calculated using these $z$-scored rates. For all cells with an increase in activity in response to the stimulus, an AROC $>0.5$ indicated reliably higher rates for the most preferred speed. A subset of DLPFC neurons showed a decrease in activity during stimulus presentation. For these suppressive neurons, AROC $>0.5$ indicated reliably lower rates for the most preferred speed. The significance of each analysis window was established by a permutation test, randomly redistributing firing rates for all the trials into most and least preferred speed groups, regardless of the actual S1 or S2 speeds (Efron and Tibshirani, 1993). An AROC was then calculated from the redistributed groups, and the process was repeated 1000 times, creating a distribution AROCs. The actual AROC value was deemed significant if it fell in the top or bottom $2.5 \%$ of the distribution $(p<$ 0.05 , two-tailed test).

Speed tuning. Speeds used during each recording session were selected to bracket each animal's behavioral threshold. For all neurons, $z$-scored responses to each presented speed were fit with a log-Gaussian function (eq 1) (Nover et al., 2005):

$$
\begin{gathered}
\mathrm{R}(\mathrm{S})=\mathrm{R}_{0}+\mathrm{A} \times \exp \left[-\frac{\log [\mathrm{q}(\mathrm{S})]^{2}}{2 \sigma^{2}}\right] \\
\mathrm{q}(\mathrm{S})=\frac{\mathrm{S}+\mathrm{S}_{0}}{\mathrm{~S}_{\mathrm{p}}+\mathrm{S}_{0}}
\end{gathered}
$$

where $\mathrm{R}_{0}, \mathrm{~A}, \mathrm{~S}_{\mathrm{o}}, \mathrm{S}_{\mathrm{p}}$, and $\sigma$ are free parameters. The parameters $R_{0}$ and $A$ correspond to the baseline activity and depth of modulation. Preferred speed and tuning width are given by $S_{p}$ and $\sigma$, respectively. $S_{0}$ is an offset parameter to prevent the function from becoming undefined at very low preferred speeds. $R^{2}$ values were obtained from each fit and used as a comparative goodness-of-fit measure. Based on the peak of the best-fit $\log$-Gaussian function cells with $R^{2}$ values $>0.6$ were categorized into one of three groups: "high pass" cells preferring higher speeds $\left(>16^{\circ} / \mathrm{s}\right)$; "low pass" cells preferring lower speeds $(<5 \%$ ), and "bandpass" cells preferring intermediate speeds $(5-16 \%$ s) (Fig. $4 B$ ).

\section{Analysis of delay activity}

Incidence of neurons active during the delay. We performed a running significance test comparing activity in the delay to baseline levels com- 
puted for a $200 \mathrm{~ms}$ window $500 \mathrm{~ms}$ before $\mathrm{S} 1$ onset $(p<0.05$, Wilcoxon signed-rank test). This analysis was performed in nonoverlapping $100 \mathrm{~ms}$ windows stepped through the delay for all neurons.

Time-dependent modulation Many neurons showed increases or decreases in activity with time in delay. To evaluate these time-dependent changes, we computed a Delay Modulation Index (DMI), which compared firing rates (FR) in a $200 \mathrm{~ms}$ window in the middle of the delay $(1250 \mathrm{~ms})$ with activity at the end of the delay $(1900 \mathrm{~ms})(\mathrm{DMI}=$ $\left[\mathrm{FR}_{(1900)}-\mathrm{FR}_{(1250)}\right] /\left[\mathrm{FR}_{(1900)}+\mathrm{FR}_{(1250)}\right)$. For this index, positive values were indicative of increasing activity whereas negative values indicated decreasing activity. Significance of individual DMIs was evaluated by a Wilcoxon signed-rank test at $p<0.01$. Differences in the median DMI between the two cell types were assessed by a Mann-Whitney $U$ test $(p<0.05)$.

Speed-selective activity. We used ROC analysis to compare the activity after the two most different speed values (fastest vs slowest) presented during S1. The speed that corresponded to the highest activity in the first significant epoch was defined that neuron's "most preferred speed" and the other that neuron's "least preferred speed." Durations of all speedselective epochs were determined with a sliding significance test in a 100 $\mathrm{ms}$ window stepped in $10 \mathrm{~ms}$ intervals. The onset of a significant epoch was taken as the center of the first significant window of at least 7 consecutive significant windows. The offset of an epoch was taken as the center of the first of at least 7 consecutive nonsignificant windows. The duration was calculated as the difference between onset and offset times.

\section{Responses during S2}

Speed selectivity. Speed selectivity during S2 was quantified with ROC analysis, as described above (in Speed selectivity) for S1. The "most preferred" and "least preferred" speeds were determined independently of those determined for responses to S1.

Comparison effects. The speed discrimination task contained two types of randomly interleaved trials: trials with S1 and S2 moving at the same speed (S-trials) and trials with S1 and S2 moving at different speeds (D-trials). To determine the effect of speed during $\mathrm{S} 1$ on responses during S2, we compared responses to the same S2 on S- and D-trials. Because of the design of our task, the base speed ( 2 or $4 \%$ s) was presented more frequently than any other speed. For this analysis, we used all trials containing S2 moving at base speed in either the preferred or antipreferred direction. S2 responses for the two directions were $z$-scored and combined to form two distributions of activity: one for S-trials and one for D-trials and used ROC analysis to compare responses during the two types of trials. Cells with AROC values $>0.5$ showed stronger responses, and thus, a preference for S-trials, whereas cells with AROC $<0.5$ showed stronger responses on D-trials. To identify periods of significant differences between these two types of trials, we used a $100 \mathrm{~ms}$ window slid across spike trains in $10 \mathrm{~ms}$ steps. To categorize responses as preferring S- or D-trials, we adopted criterion values of $>0.65$ and $<0.35$ during at least 7 consecutive bins. Neurons with a sufficient number of consecutive periods with values $>0.65$ were categorized into the $S>D$ group (same $>$ different), whereas neurons meeting the $<0.35$ consecutive criterion formed a $\mathrm{D}>\mathrm{S}$ group (different $>$ same). If a neuron showed activity satisfying both criteria, the effect with the longest duration determined categorization. To determine whether the differences between S- and D-trials depend on the differences in speed between $\mathrm{S} 1$ and S2, AROC values were computed separately for each level of speed difference used during the task.

To compare directly the magnitude and timing of $\mathrm{S}>\mathrm{D}$ and $\mathrm{D}>\mathrm{S}$ effects, AROC values were recalculated, taking into account an individual neuron's preferred trial-type ("same" or "different"). In this analysis, AROC values $>0.5$ indicate greater activity during the preferred trialtype of a neuron (S- or D-trials). Conversely, an AROC $<0.5$ corresponds to weaker activity during a neuron's preferred trial type. This transformation had the effect of reflecting $\mathrm{D}<\mathrm{S}$ neurons above 0.5 .

Choice probability $(C P)$. We also examined whether activity during the S2 and post-S2 periods was predictive of the choices monkeys made on each trial. Because the monkeys sometimes reported S-trials as "different" and sometimes as "same," choice-related activity could be computed by comparing firing rates on trials with identical sensory conditions (e.g., S1 and S2 moving at $4 \%$ s) but different behavioral reports. A similar analysis was used in preceding reports (Zaksas and Pasternak, 2006; Lui and Pasternak, 2011; Hussar and Pasternak, 2012) and is similar to the approach introduced by (Britten et al., 1996). Only cells with at least five S-reports (right button) and five D-reports (left button press) were included in this analysis. Spike counts during trials with sufficient right (same) and left (different) reports were $z$-scored to account for any differences in activity related to the two possible S2 directions. These $z$-scored spike counts were then combined, creating a distribution of S2 spike counts associated with S- and D-reports. ROC analysis was used to evaluate the differences between the two distributions. CP values $>0.5$ are indicative of higher activity before neuron's preferred report ("same" or "different"). To identify periods of reliable $\mathrm{CP}$, we slid a $100 \mathrm{~ms}$ window in $10 \mathrm{~ms}$ steps and used criterion values of $\geq$ 0.65 during seven consecutive bins to categorize neurons as either $\mathrm{S}$ or D-report neuron, dependent upon which report showed a greater preceding response. Significance for calculated CPs were evaluated with a bootstrap test $(p<0.05)$.

\section{Results}

We recorded the activity of 150 task-related PFC neurons in two animals (Monkey 1, $n=87$; Monkey 2, $n=63$ ) during the performance of a speed discrimination task (Fig. 1A). These neurons were further subdivided into NS $(n=37)$ and BS $(n=113)$ based upon the duration of their mean action potentials (see Materials and Methods). The average psychometric functions for both monkeys, based on 30,142 trials collected during 150 recording sessions, are shown in Figure $1 B$. The performance of both monkeys decreased with smaller differences between the two comparison stimuli. Overall, Monkey 1 was more accurate than Monkey 2 (speed difference thresholds: Monkey 1, $0.85 \pm 0.04$; Monkey 2, $1.48 \pm 0.01)$. Whereas Monkey 1 reliably discriminated between stimuli moving at 4 and $7.4^{\circ} \mathrm{s}$, Monkey 2 needed a larger difference in speed to reach $75 \%$ correct performance ( $4 \mathrm{vs} 10 \%$ s).

\section{Patterns of speed-selective activity recorded during the task} Figure 2 shows the activity of two example neurons, NS (top) and BS (bottom), recorded during the speed comparison task. The response of the NS cell was strongest to the stimulus moving at $4 \%$, decreasing at higher speeds. This can be seen in Figure $2 C$, showing an average response to each speed presented during $\mathrm{S} 1$ (left plot) and S2 (right plot). During the delay, this neuron showed relatively weak activity with small and transient differences in firing rates associated with speeds presented during S1. The BS cell (Fig. 2B) also showed a speed-dependent response during $S 1$ and S2, although it preferred higher speeds. This preference can be seen in Figure $2 D$, plotting average response as a function of speed during both S1 and S2. These two example cells highlight some of the patterns characteristic of many neurons recorded during this task, including speed-selective responses during S1 and S2 in both NS and BS cells, and relatively modest speed-related differences in delay activity.

\section{Speed-selective responses}

We previously reported that during the direction discrimination task both BS and NS neurons were equally likely to reliably represent motion direction (Hussar and Pasternak, 2009, 2012). Here, we examined whether both cell types also represent stimulus speed. We focused this analysis on responses during S1 because we found that stimulus selectivity during S1 and S2 was similar. Because of the relatively limited range of speeds used in the comparison task, it was often not possible to match each neuron's speed preference. Thus, we identified speeds as the 


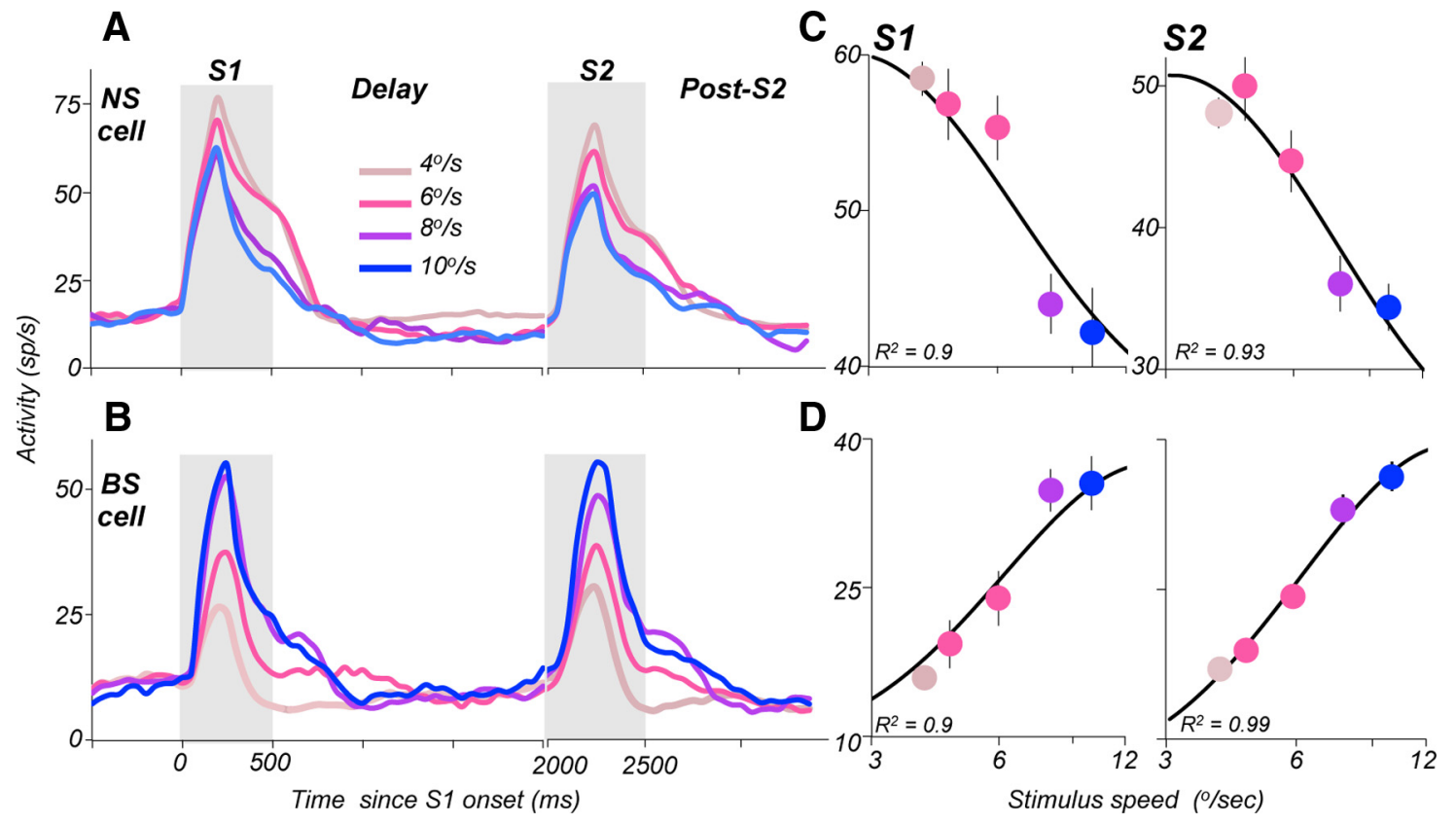

Figure 2. Speed-selective activity during speed discrimination task. $A, B$, Spike density functions for an example NS putative interneuron $(\boldsymbol{A})$ and $B S$ putative pyramidal ( $\boldsymbol{B}$ ) neuron. Spike trains were convolved with a $20 \mathrm{~ms}$ Gaussian to show activity. Different colored lines represent average firing rates for each speed. Both neurons were DS. The data are shown for S1 and S2 moving in the neuron's preferred direction. $\boldsymbol{C}, \boldsymbol{D}$, Response tuning to speeds presented during S1 (left) and S2 (right) for the NS ( $\boldsymbol{C}$ ) and BS ( $\boldsymbol{D}$ ) cells shown in $\boldsymbol{A}$ and $\boldsymbol{B}$. Responses were computed for activity measured during 50 -500 ms after stimulus offset. The curve fitting the data is the best-fit log-Gaussian function for each neuron (Materials and Methods).

"most preferred" and "least preferred," with the understanding that the two terms did not necessarily describe the most and least optimal speeds for a given neuron. To categorize individual neurons as speed-selective, we performed a sliding ROC analysis using a $100 \mathrm{~ms}$ window stepped $100 \mathrm{~ms}$ throughout the stimulus presentation. Any neuron with a significant difference in activity between the most preferred and least preferred speeds was classified as speed-selective (bootstrap test, $p<0.05$ ). Using this criterion, $63 \%(n=95)$ of neurons were classified as speed-selective (NS: $65 \%, n=24$; BS: $63 \%, n=71$ ). Figure $3 A$ shows the average normalized response to the most preferred (solid line) and least preferred (broken line) speeds for all NS (left plot) and BS (right plot) neurons. Speed selectivity in these two groups of neurons, plotted in Figure $3 B, C$, shows that both cell types reliably distinguished between differences in stimulus speed, although stimulus selectivity in NS neurons was slightly stronger (Fig. 3C, 50-500 $\mathrm{ms}, \mathrm{NS}=0.70 \pm 0.004$, BS $=0.65 \pm 0.007, p=0.036$, MannWhitney $U$ test). NS neurons also showed a more rapid onset of selective activity than BS neurons (NS = $150 \pm 34 \mathrm{~ms}$, BS = $225 \pm 46 \mathrm{~ms} ; p=0.04$, Mann-Whitney $U$ test).

We were interested whether speed-selective neurons during the speed task were also DS during the direction task. We were able to address this question because the majority of these neurons were recorded under both tasks conditions (Hussar and Pasternak, 2009, 2012), allowing a direct comparison of selectivity for direction and speed in the same neurons. To determine DS of each neuron, we used sliding ROC analysis, identical to that used to determine speed selectivity. Any neuron with a significant difference in activity at any time was considered DS (bootstrap test, $p<0.05$ ). The analysis of all speed-selective neurons with a paired direction discrimination recording $(n=$ $77)$ revealed that $86 \%(n=66)$ of speed-selective neurons were also selective for direction, and this result was similar across cell types (NS: $84 \%, n=16$; BS: $86 \%, n=50$ ). The pronounced overlap of this selectivity in individual neurons is similar to what has been found in MT (Maunsell and Van Essen, 1983; Rodman and Albright, 1987; Lagae et al., 1993), providing additional evidence for MT as a likely source of motion information in DLPFC.

If speed selectivity in the DLPFC is inherited from the motionprocessing neurons residing in MT, it is likely that its tuning may also be similar. Previous studies revealed a range of speed preferences in MT, with cells preferring slow, intermediate, or fast motion (Maunsell and Van Essen, 1983; Liu and Newsome, 2005) and their tuning was well characterized by a log Gaussian function (Nover et al., 2005). To examine whether speed tuning in the DLPFC resembled that of MT, we used a log-Gaussian function to fit responses of all neurons categorized as speed-selective $(n=$ 95). The distribution of $R^{2}$ values for these fits, plotted in Figure $4 A$, shows that the majority of NS and BS neurons were well fit by the log-Gaussian function $\left(R^{2}>0.6\right.$; NS $=58 \%, n=14$; BS $=$ $60 \%, n=57)$, and the quality of fits was similar between the two cell types (median $R^{2}$ value, NS $=0.73 \pm 0.062$; $\mathrm{BS}=0.7 \pm$ $0.034 ; p=0.82$, Mann-Whitney $U$ test). We used neurons with $R^{2}>0.6$ to categorize speed tuning as low-pass, bandpass, or high-pass (Fig. 4B; see Materials and Methods). This analysis revealed a higher proportion of cells preferring slower $(46 \%)$ than higher speeds $(31 \%)$, and an even smaller number of neurons with preferences for intermediate speeds $(23 \%)$. We should note that, because of the behavioral constraints, the range of presented speeds was probably too narrow to fully characterize tuning in many neurons. For that reason, it is likely that our measurements may be overestimating the number of neurons preferring higher and lower speeds and underestimating the number of cell with bandpass properties. Nevertheless, our data show that many DLPFC neurons exhibited speed-selective responses and that this selectivity could be adequately characterized by a log-Gaussian function, similar to speed selectivity recorded in area MT (Nover et al., 2005). 

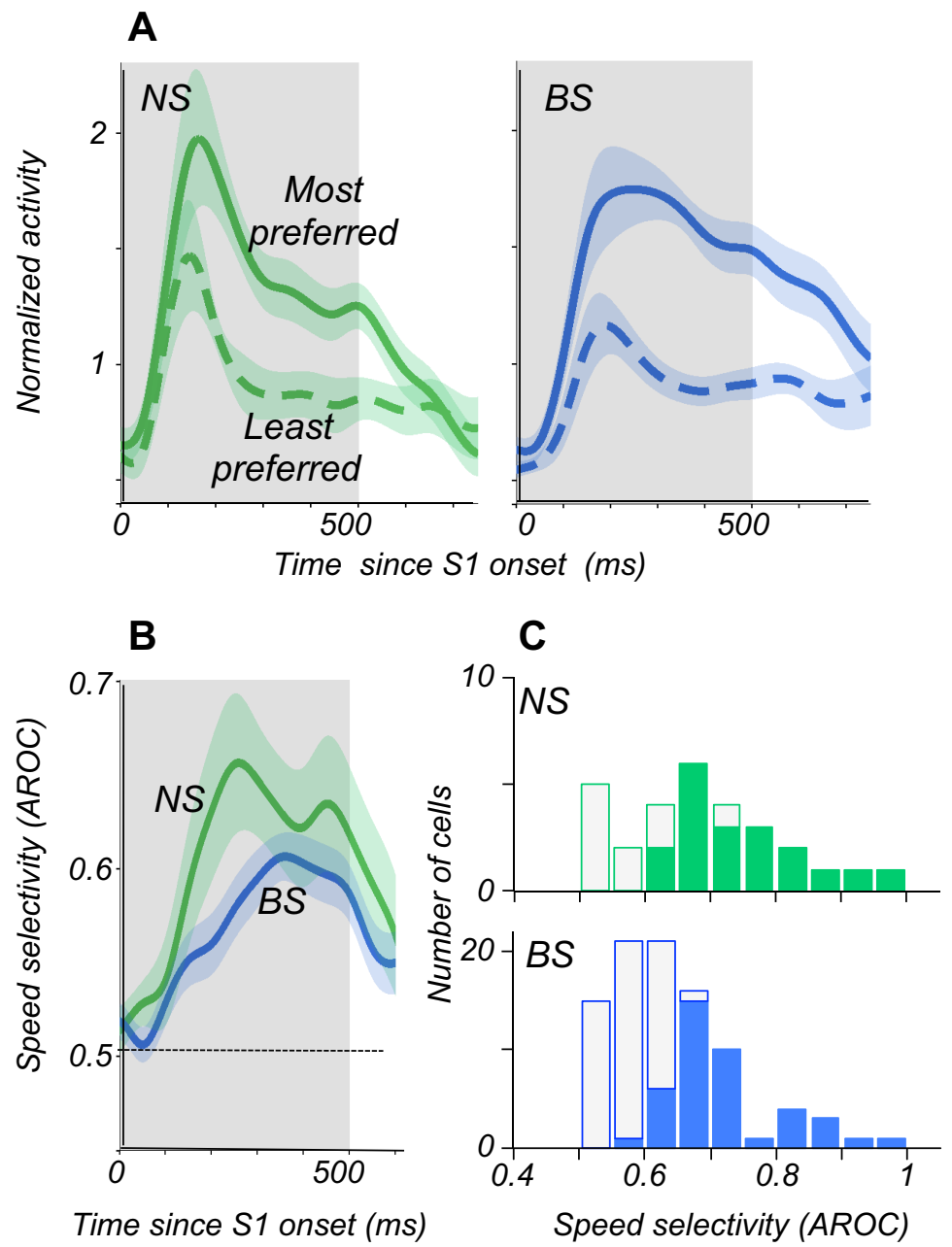

Figure 3. Response selectivity of NS and BS neurons for stimulus speed. $A$, Average normalized response to $S 1$ for the most preferred (solid line) and least preferred (broken line) stimulus speeds for all recorded NS (left plot, $n=35$ ) and BS (right plot, $n=$ 97). $\boldsymbol{B}$, Average speed selectivity (AROC) for NS and BS neurons computed by comparing responses shown in $\boldsymbol{A}$. $\boldsymbol{C}$, Distribution of $A R O C$ for all NS (top) and BS (bottom) neurons shown in $B$. AROCs were calculated for the 50-550 ms period after the onset of $S 1$. Neurons with significant selectivity $(p<0.05)$ are indicated by filled colored bars. The distributions show stronger average selectivity in NS neurons (NS $=0.70 \pm 0.004, \mathrm{BS}=0.65 \pm 0.007, p=0.036$, Mann-Whitney U test).

\section{Speed selectivity is modulated by task demands}

Prevalence of speed selectivity in the DLPFC and its similarity to responses recorded in MT raise the question whether this selectivity is a reflection of their specialization for representing stimulus speed or emerges as a result of its behavioral relevance. We addressed this question by manipulating task demands while keeping the sensory conditions the same and comparing response selectivity of individual neurons during the speed task to that recorded during the passive fixation task (Fig. $5 A$ ). During this task, the animals were rewarded for initiating a trial and maintaining fixation throughout the presentation of the two motion stimuli. The comparison was performed on responses of a subset of speed-selective neurons with sufficient number of trials in both tasks during S1 $(n=30)$ and during S2 $(n=33)$. Figure $5 B$ shows speed selectivity of S1 and S2 responses during the two tasks. Because of the similarity in the behavior of NS and BS neurons during passive fixation (Hussar and Pasternak, 2009), the data for the two cell types were combined. Reduced selectivity of each cell type can be seen in corresponding scatterplots (Fig. $5 C)$ and was observed during both S1 and S2 $(p<0.05$, Wilcoxon sign-rank test). We should note that the decrease in speed selec- tivity was unlikely to be the result of weakening of responses during passive fixation as the change in firing rates, although significant, was relatively modest (speed task, $21.8 \mathrm{sp} / \mathrm{s}$, passive fixation, $18.8 \mathrm{sp} / \mathrm{s}$; $p=0.019$; Wilcoxon). We quantified the reduction in selectivity by computing a Task Effect Index $=$ AROC $_{(\text {passive })}-$ $\mathrm{AROC}_{\text {(speed) (Hussar and Pasternak, }}$ 2009). The average Task Effect Index, shown separately for S1 and S2 across time (Fig. 5D), illustrates the larger loss of speed selectivity during S2. This difference in the task induced change in response selectivity during S1 and S2 is well illustrated on a cell-by-cell basis (Fig. 5E). This observation parallels previously reported asymmetries in task effects between S1 and S2 during direction discrimination (Hussar and Pasternak, 2009; Hussar and Pasternak, 2010). These asymmetries most likely reflect differences in task demands during S1 and S2. The onset of S1 indicates successful initiation of a trial and the requirement to identify and commit to memory stimulus speed. During S2, in addition to identifying the stimulus, subjects must also retrieve the remembered speed, compare it with the current stimulus, and make a decision. During passive fixation, on the other hand, while the S1 onset still signals trial initiation, additional task demands during S2 are removed, providing an explanation for the greater reduction in activity and selectivity during S2. High trial-to-trial variability recorded during S2, but not during S1, during a passive fixation task further supports this interpretation (Hussar and Pasternak, 2010). Overall, these results highlight the ability of DLPFC neurons to filter incoming sensory information based on its behavioral relevance.

\section{BS neurons are more likely to carry anticipatory activity during the delay}

In our task, the length of the delay was held constant; therefore, the time of S2 onset was highly predictable. We examined whether the activity changed with time in delay, reflecting the predictable structure of our task. As an initial measure of delay activity, we performed a running significance test comparing activity in delay to baseline levels $(p<0.05$, Wilcoxon signed-rank test). The results of this analysis are shown in Figure 6A. Early in the delay, immediately after the offset of $\mathrm{S} 1$, a similar proportion of NS and BS neurons showed significant activity $\left(\chi^{2}, p>0.05\right)$, most likely reflecting the still present response to S1. However, after the first half of the delay, the incidence of NS neurons with delay activity leveled off at $\sim 30 \%$, whereas the proportion of BS neurons showed a steady increase, resulting in a greater percentage of active BS neurons during the last $400 \mathrm{~ms}$ of the delay (Fig. $6 A, \chi^{2}, p=0.037$ ), suggesting a unique role for these neurons in preparation for the upcoming S2 (Hussar and Pasternak, 2010; Hussar and Pasternak, 2012). 
A

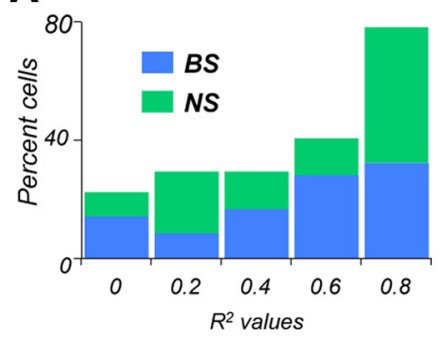

B

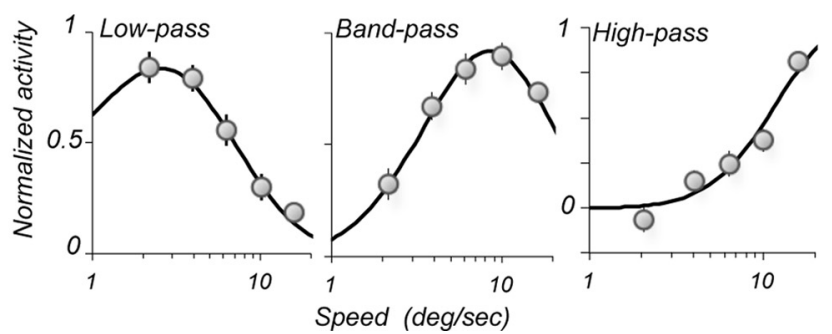

Figure 4. Speed tuning functions. $\boldsymbol{A}$, Distribution of $R^{2}$ values for $\log$-Gaussian fits of all significantly speed-selective NS and BS neurons $(n=95)$. $R^{2}>0.6$ was defined as well fit ( $n=57$ ), whereas $R^{2}<0.6$ was labeled as not well fit $(n=38)$. $B$, Types of speed tuning encountered in the PFC. Neurons with low pass $(n=26)$, bandpass $(n=13)$, and high-pass $(n=18)$ speed tuning. Only cells that were well fit by the log-Gaussian function $\left(R^{2}>0.6\right)$ were used for this analysis. Individual data points represent average responses to each speed recorded during the period of 50-500 ms after $\$ 1$ onset. Black lines indicate the average fit for all low-pass, bandpass, and high-pass neurons.

To examine the apparent time-dependent changes throughout the delay we calculated DMI (see Materials and Methods) for all NS and BS cells. This index compared activity between the middle and late delay, with positive and negative values indicating increasing and decreasing firing rates, respectively. The distributions of the DMIs for all NS and BS neurons are shown in Figure $6 B$, with neurons with significant effects indicated by colored columns (Mann-Whitney $U$ test, $p<0.01$ ). The distributions show that with time in delay some neurons increased firing rates $(\mathrm{DMI}>0)$, some showed decreased rates $(\mathrm{DMI}<0)$ and a subset of neurons showed no significant change (gray columns). While the incidence of delay modulation in the two types of neurons was similar (NS $=51 \%, \mathrm{BS}=59 \%, p=0.4, \chi^{2}$ test), the absolute strength of this modulation was significantly greater in BS neurons than NS neurons $(\mathrm{DMI}=0.22 \pm 0.019$ vs $\mathrm{DMI}=$ $0.13 \pm 0.02$; Mann-Whitney $U$ test, $p=0.007$ ).

These data illustrate pronounced differences in delay activity between the two groups of cells, with BS putative pyramidal cells showing stronger modulation before S2 onset. These results parallel those observed during the direction discrimination task (Hussar and Pasternak, 2012), providing further support for the role for BS neurons in the anticipation of salient trial events (Hussar and Pasternak, 2010).

To determine whether the observed change in delay activity reflects preparation for the upcoming comparison the animal had to perform during S2, we examined delay activity during the passive fixation task, where no such comparison was required (Fig. $5 A$ ). Figure $7 A$ shows the average delay activity for neurons with increasing delay activity $(n=21)$ during the active discrimination task (solid line) and during passive fixation (broken line). While these averages are based on combining data from both cell types, changes in activity in individual BS and NS neurons can be seen in the scatterplot in Figure $7 B$. Overall, DMI was significantly weaker during the passive fixation task ( $p=0.00009$, Wilcoxon sign-rank test) and this effect was similar for cells with increasing and decreasing firing rates with time in delay ( $p=$ 0.81 , Mann-Whitney $U$ test). This result parallels the decrease in delay modulation during passive fixation compared with the direction discrimination task (Hussar and Pasternak, 2012), providing further support for the interpretation that gradual changes in firing rates with time in delay are most likely preparatory in nature, reflecting the neuron's engagement in the task (Hussar and Pasternak, 2010).

\section{Speed-selective activity during the delay}

Our data revealed the preparatory nature of delay activity of many DLPFC neurons. Because the monkey's task was to remem- ber stimulus speed, we examined whether, in addition to the timing signals, delay activity also carried information about the preceding speed. We quantified stimulus selectivity during the delay with ROC analysis by comparing the activity following the slowest and the fastest speeds presented during $\mathrm{S} 1$ by sliding a $200 \mathrm{~ms}$ window across the trial. Figure $8 A$ shows the results for all BS $(n=113)$ and NS $(n=37)$ neurons. AROC values were sorted by the average deviation from 0.5 during the $S 1$ response. Dark blue colors correspond to greater activity for the speed preference during S1 (either the slowest or the fastest speed). If a given neuron's preference reversed later in the trial, it was indicated by AROC $<0.5$ (dark red colors). The data show that, during the delay, periods of speed selectivity were reliable but relatively short. This was quantified by determining durations of all selective epochs by sliding across the delay a $100 \mathrm{~ms}$ window in $10 \mathrm{~ms}$ steps ( $p<0.05$, Wilcoxon sign-rank test). The distribution of these durations (Fig. $8 B$ ) shows that the periods of selectivity for both cell types were brief, on average not exceeding $400 \mathrm{~ms}$ (NS = $379 \mathrm{~ms} \pm 41.6$; $\mathrm{BS}=388 \mathrm{~ms} \pm 31.5 ; p=0.87$, Wilcoxon signedrank test). Note that the majority of longer duration epochs ( $>500 \mathrm{~ms} ; n=31$ ) belonged to BS neurons (BS, 74\%, $n=23$; NS, $26 \%, n=8)$.

Although in individual neurons delay selectivity was transient and appeared at different times, when combined this activity could provide a continuous representation of the preceding S1. To determine the overall speed selectivity in the recorded sample of NS and BS cells, we calculated rectified AROC by reflecting the values above 0.5 (Fig. $8 C$ ). Although this calculation allowed us to estimate the overall speed selectivity discounting its sign, it resulted in elevating baseline AROC values to 0.55 (indicated by a dotted black line in Fig. 8C). During S1, each cell type exhibited pronounced speed tuning, with NS neurons showing slightly greater selectivity ( $p<0.05$, Mann-Whitney $U$ test). This selectivity was also comparable during the early and middle delay. However, in the last $500 \mathrm{~ms}$, speed selectivity in the BS cells began to increase, most likely reflecting the greater proportion of these neurons showing higher activity and a larger number of speed-selective epochs (Figs. $6 A$ and $8 A$ ), leading to greater delay selectivity in BS $(p<0.05$, Mann-Whitney $U$ test $)$. These data show that, while in individual neurons stimulus selectivity was transient, the information about the preceding S1 appears to be distributed among neurons and represented largely by BS neurons. This finding parallels our recent observation of DS delay activity recorded during the direction task (Hussar and Pasternak, 2012).

We also found that in individual neurons delay selectivity did not depend on their response selectivity during S1. The data in 
A

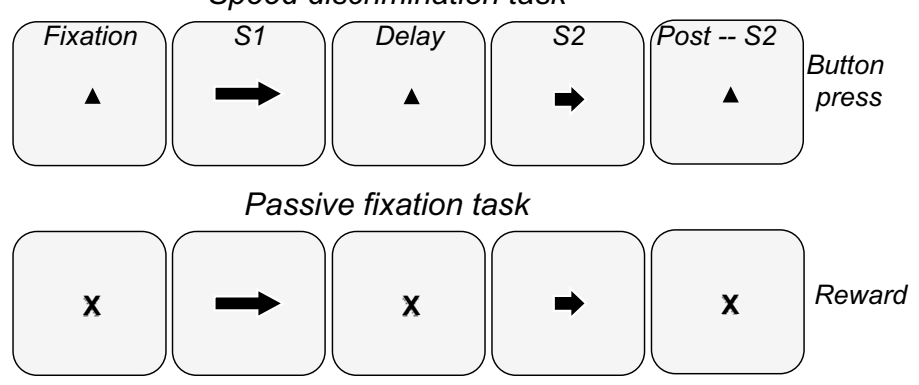

B
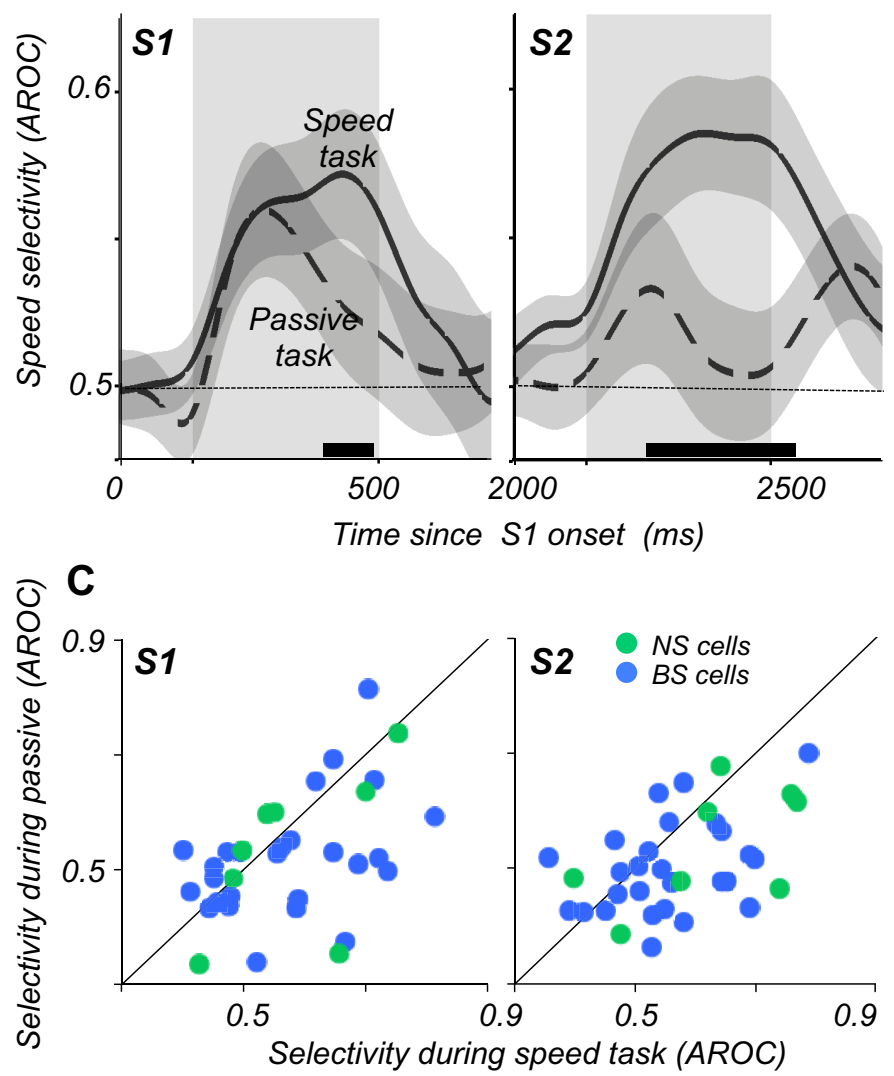

D

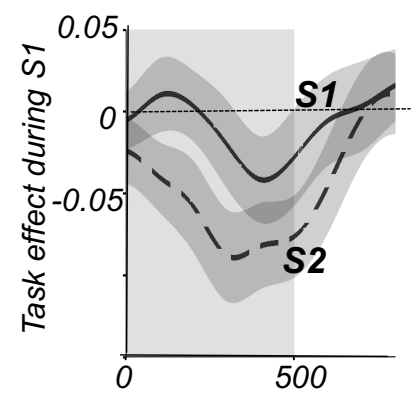

Time since stimulus onset (ms)

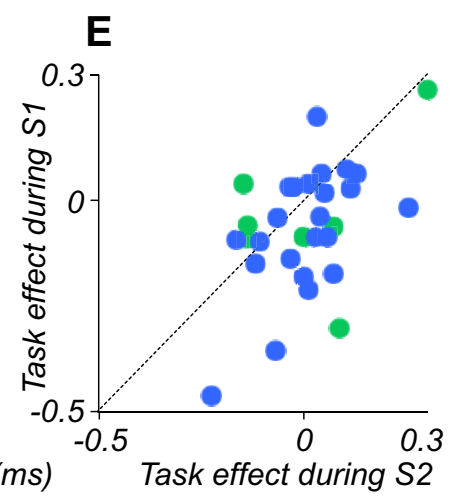

Figure 5. Reduced speed selectivity during passive fixation. $\boldsymbol{A}$, Stimulus conditions during speed discrimination and passive fixation. Sensory conditions during the two tasks were identical, except for different fixation targets ( $\boldsymbol{\Delta}$ vs X). During the passive fixation task, the monkeys were rewarded at the end of each trial and were not required to press response buttons. $\boldsymbol{B}$, Speed selectivity of responses to $S 1(n=30)$ and $S 2(n=33)$ recorded during the two tasks. Thick black lines along $x$-axis indicate times of significant differences between the two curves ( $p<0.05$, Wilcoxon sign-rank test). $C$, Selectivity of individual NS and BS neurons during the two tasks. Each data point represents activity recorded during the last $100 \mathrm{~ms}$ of the response ( $400-500 \mathrm{~ms})$. Speed selectivity was weaker on passive fixation trials during $\mathrm{S} 1(p<0.012)$ and $\mathrm{S} 2(p<$ 0.002). D. Comparison of task effects on selectivity during S1 and S2. Task effect was computed as the difference in AROC
Figure $8 A$ illustrate that many cells with speed-selective responses during $S 1$ (blue traces) exhibited little speed selectivity during the delay (the absence of blue or red traces) and many cells with selectivity during the delay (blue traces or red traces) did not show selectivity during S1. This pattern suggests that the selectivity displayed by individual neurons during delay may be independent of their response selectivity during S1. We quantified this observation by directly comparing selectivity recorded during $\mathrm{S} 1$ and during three periods of the delay. We limited this analysis to neurons with significant selectivity during S1 $(p<0.05)$ and on a cell-by-cell basis compared it with the selectivity recorded during each of the three consecutive delay periods: early (500-1000 ms), middle (1000-1500 ms), and late (1500$2000 \mathrm{~ms}$ ) (Fig. 8D). In all three graphs, open symbols show cells with no reliable delay selectivity (AROC $\sim 0.5$ ), whereas filled symbols show cells with significant delay selectivity $(p<0.05)$. The filled data points in the upper half of each graph (AROC > 0.5) indicate significant delay selectivity of the same sign as during S1. Filled data points in the lower half of each plot indicate significant selectivity opposite to that of S1 (AROC < 0.5). The bar plots on the right are summaries, showing the proportion of cells preferring the same (upper columns) or opposite (lower columns) speeds as during S1 and cells with no significant delay selectivity (middle columns). In all three epochs, approximately comparable numbers of neurons showed delay selectivity that was either consistent (AROC $>0.5$ ) or inconsistent $($ AROC $<0.5)$ with stimulus driven selectivity. This shows a clear disassociation between speed preferences during S1 and during the delay.

The nature of this stimulus selective delay activity raises the question whether the signals it carries are used during the task. We addressed this question by comparing selectivity of the same neurons during the speed task and during the passive fixation task. Selectivity preferences were identified during the speed task for all neurons, except those that only showed

\section{$\leftarrow$}

values during the two tasks ( $A R O C_{\text {passive }}-A_{R O C}$ speed $)$. Negative values indicate a decrease in selectivity during passive fixation. $\boldsymbol{E}$, Cell-by-cell comparison of task effects during S1 and S2. Each data point represents task effect measured at 100-300 ms after stimulus onset. During passive fixation, selectivity loss was greater during $\mathrm{S} 2$ ( $p=0.0175$, Wilcoxon sign-rank test). 

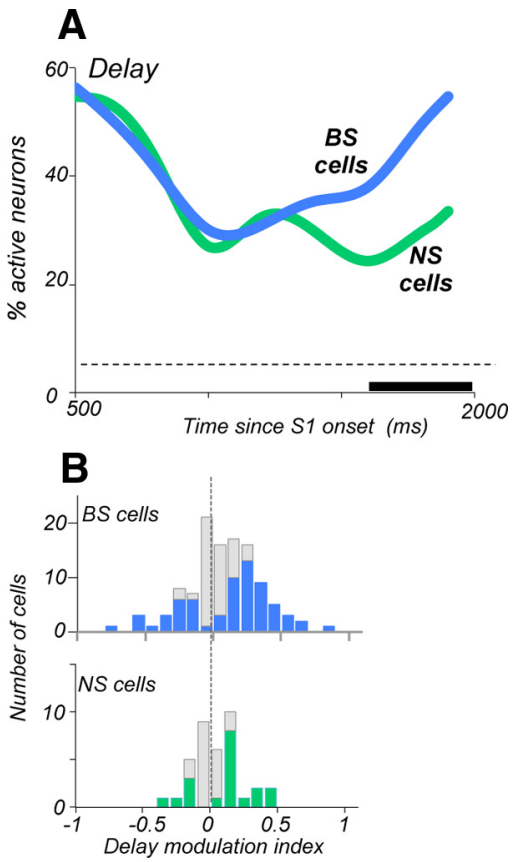

Figure 6. Time-dependent modulation of delay activity. $\boldsymbol{A}$, Incidence of neurons with significant delay activity. The thick black line indicates the period of significant difference between NS and BS $\left(\chi^{2}\right.$ test, $\left.p<0.05\right)$. The dashed line at $5 \%$ indicates the level of significance expected by chance. NS cells, $n=37 ; B S, n=113$. $B$, Time-dependent modulation of delay activity for $B S$ (top) and NS (bottom) neurons. DMI = (activity late delay) - activity middle delay)/(activity late delay + activity middle delay). Late delay, last $200 \mathrm{~ms}$ of delay; middle delay, $200 \mathrm{~ms}$ centered at $1250 \mathrm{~ms}$. Values $>0$ indicate higher activity in late delay; values $<0$ indicate lower activity in late delay. Filled colored bars, cells with significant modulation (Wilcoxon sign-rank test, $p<0.01$ ); gray bars, cells with no delay modulation. $\mathrm{BS}$ neurons showed stronger delay modulation (NS, DMI $=0.126 \pm 0.02 ; \mathrm{BS}, \mathrm{DMI}=0.22 \pm 0.02$; Mann-Whitney $U$ test, $p=0.011)$.

significant selectivity during passive fixation (square markers). The data recorded during the two tasks with identical sensory conditions was available for a relatively small subset of neurons $(\mathrm{NS}=7, \mathrm{BS}=27)$. The results, plotted in Figure $9 \mathrm{~A}$, show that during passive fixation the average speed selectivity was substantially reduced throughout the delay. Figure $9 B$ shows a direct cell-by-cell comparison of selectivity in the three consecutive 500 ms delay periods during the two tasks. Throughout the delay, representation of speed during active discrimination was greater than during passive fixation (early delay, $p=0.005$; middle delay, $p=0.003$; late delay, $p=0.0001$, Wilcoxon sign-rank test). This effect is consistent with decreased speed selectivity during $S 1$ and S2 (Fig. 5) and reminiscent of the loss of DS delay activity recorded during the direction discrimination task under passive fixation (Hussar and Pasternak, 2012). This reduction of delay selectivity during passive fixation suggests that this selectivity may be utilized during active discrimination tasks.

\section{Modulation of S2 responses by the preceding speed}

During S2, the animals must perform a comparison between the preceding (S1) and current speed (Fig. 10A). We examined whether responses during $\mathrm{S} 2$ reflect this process by comparing such responses on S-trials (S1 and S2 move at the same speed) and on D-trials (S1 and S2 moved at different speeds). Differences between responses to identical S2 stimuli on S- and D-trials would be indicative of modulation of S2 responses by the preceding $\mathrm{S} 1$, a likely reflection of a comparison process. We termed this modulation of $\mathrm{S} 2$ response by the preceding stimulus a compar-
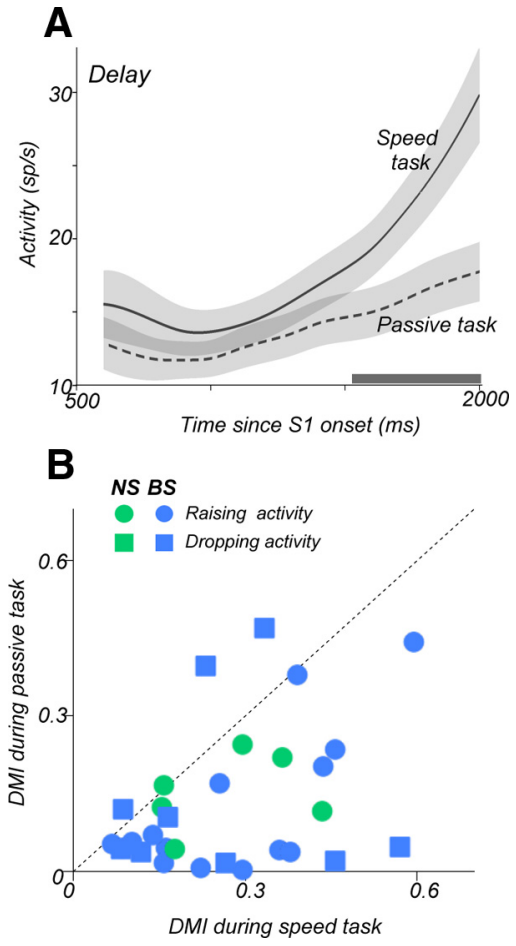

Figure 7. Reduced modulation of delay activity during passive fixation. $\boldsymbol{A}$, Comparison of delay activity during the speed and the passive fixation tasks. Only cells with significant increase in activity during the speed task were used $(n=21)$. $\boldsymbol{B}$, Cell-by-cell comparison of DMls (see Fig. 6 legend) for all NS $(n=6)$ and BS ( $n=24)$ neurons in both tasks. All negative DMls (squares) have been reflected $>0$ to facilitate the comparisons with the positive DMI (circles). Delay modulation was greatly reduced during passive fixation ( $p=0.00009$, Wilcoxon sign-rank test), with no significant difference in the size of effect between raising or decreasing activity types ( $p=0.82$, Mann-Whitney $U$ test).

ison effect (CE). Figure 10B illustrates the magnitude and sign of such effects for all recorded neurons computed for trials with maximally different speeds during S1 and S2. Cells with stronger responses on S-trials (S > D) are indicated by deep blue colors $($ AROC $>0.5)$, whereas neurons with stronger response $s$ on D-trials $(\mathrm{D}>\mathrm{S})$ by deep red colors (AROC $<0.5)$. Greenish colors correspond to responses with little or no difference between $\mathrm{S}$ - and D-trials $(\mathrm{S}=\mathrm{D})$. The data for NS and BS cells were combined because of the similarity in the incidence of these effects in the two cell groups (NS $=60 \%, \mathrm{BS}=57 \%, p=0.47, \chi^{2}$ test). Visual inspection of this figure reveals several important features. First, CEs were quite common, with the majority (59\%; $n=79$ ) showing reliable effects at some point in time during the S2/post-S2 periods. Second, with few exceptions, the two distinct trial types were represented by different and nonoverlapping groups of cells, and these cells rarely switched their preferences during or after S2. Third, neurons with greater activity on S-trials (in blue) were more common ( $>$ D, 65\%; D > S, 35\%). To facilitate the comparison between cells preferring S-trial and $D$-trials, we reflected AROCs for all $D>S$ neurons $>0.5$. The CEs for $S>D$ and $D>S$ cells recorded after the onset of S2 show a robust representation of the similarities and differences between speeds presented during S1 and S2 (Fig. 10C, D). During S2, the two types of effects showed a similar time course, both reaching their maximal effect near S2 offset (Fig. 10E). After S2 offset, the $\mathrm{CE}$ carried by neurons preferring S-trials persisted longer than the effect carried by D $>\mathrm{S}$ cells. As a result, it was more pronounced in early post-S2 period (Fig. $10 C, p<0.05$, MannWhitney $U$ test). 

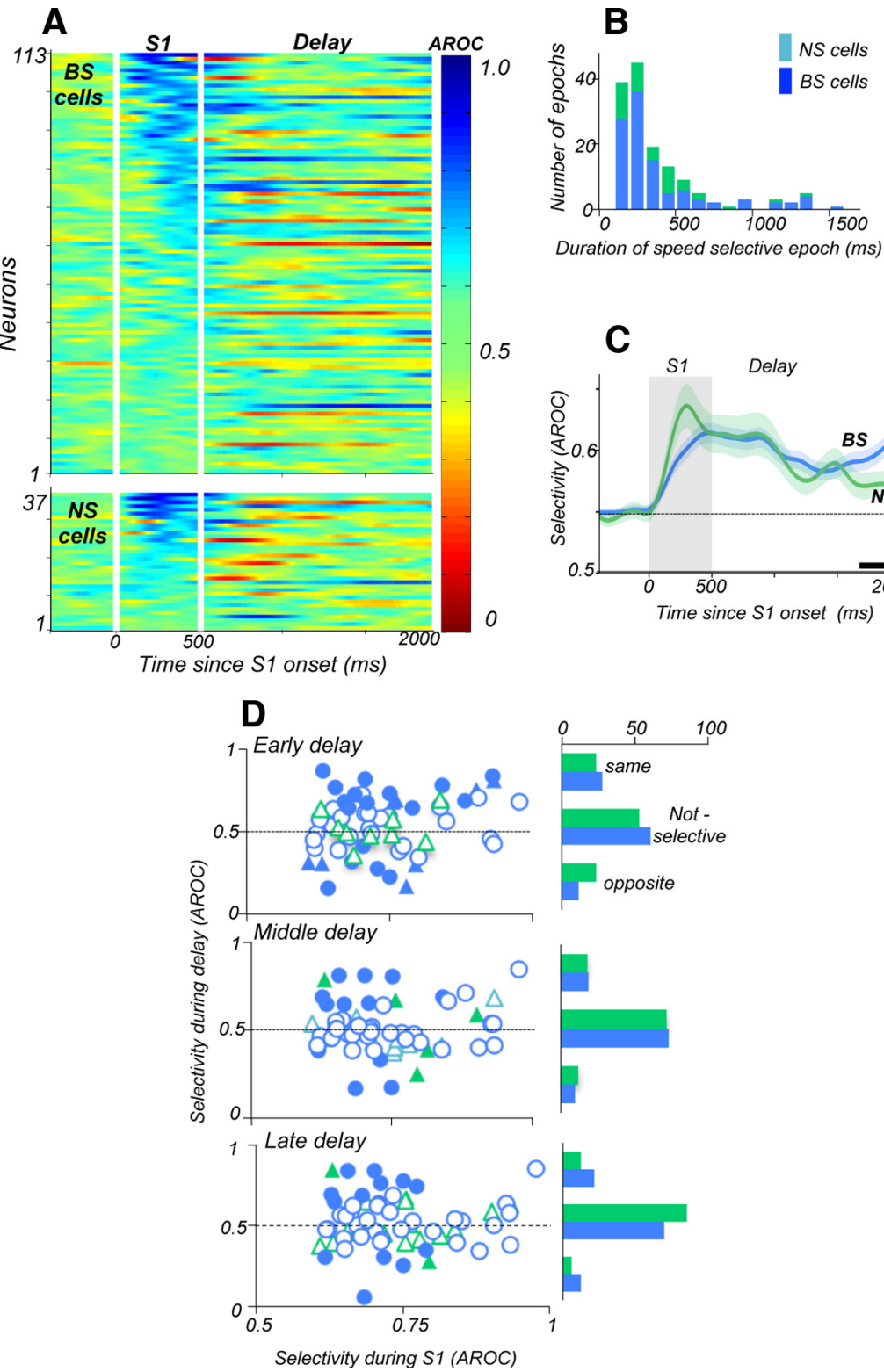

Figure 8. Speed-selective activity during the delay. $A$, Speed selectivity of individual BS $(n=113)$ and NS ( $n=37)$ neurons during $S 1$ and the delay quantified by ROC analysis. Scale bar on the right shows ROC values (most preferred speed, blue; least preferred speed, red. The data were sorted by the selectivity during $S 1(50-550 \mathrm{~ms})$. B, Durations of speed-selective epochs for NS and $B S$ neurons during the delay. $C$, Average speed selectivity for NS and BS neurons. Baseline AROC was defined by the average AROC during the last $500 \mathrm{~ms}$ of fixation. The black line along $x$-axis indicates period of significant difference between $B S$ and NS neurons (Mann-Whitney $U$ test, $p<0.05$ ). $\boldsymbol{D}$, Relationship between speed selectivity of individual neurons during $S 1$ and during the delay. Only cells with significant selectivity during $\mathrm{S} 1$ were included in this analysis (NS cells, $N=17 ; \mathrm{BS}, N=43 ; 50-550 \mathrm{~ms}$, $p<0.05$, Mann-Whitney $U$ test). Selectivity of each neuron during $S 1$ is plotted against its selectivity in early (500-1000 ms), middle (1000 - 1500 ms), and late (1500-2000 ms) delay. Filled and open symbols indicate selective $(p<0.05)$ and nonselective $(p>0.05)$ neurons, respectively, during each period of the delay. $A R O C>0.5$ indicates selectivity in the same sign as $S 1 ; A R O C<$ 0.5 indicates selectivity of opposite sign to $\mathrm{S1}$. Column plots to the right of each scatterplot show the incidence of neurons with selectivity of the same (top) or opposite (bottom) sign as that in S1. Middle columns show the incidence of nonselective cells. Note the increasing number of neurons in the middle columns with time in delay.

It can be seen in Figure $10 B$ that a small subset of cells began showing the difference between $\mathrm{S}$ - and D-trials before the appearance of S2, making it unlikely that the effect measured at that point in the trial was the result of the comparison between $S 1$ and S2. One explanation for this early occurring effect may lie in the fact that our analysis was limited to the more numerous trials in which S2 moved at the low (base) speed. Under these conditions, on S-trials S1 moved at the low speed, whereas on D-trials S1 moved at a higher speed. Thus, AROC values deviating from 0.5 in late delay could be a reflection of speed-selective delay activity bleeding into $\mathrm{S} 2$ responses. We should note that these "prematurely" appearing effects were encountered only in a subset of neurons $(24 \%, N=19)$. In the majority of cells, CEs became apparent at least $100 \mathrm{~ms}$ after the onset of S2.

Because of the statistic of our task, the base speed (either 2 or $4 \%$ ) was presented most frequently and was paired either with S1 moving at the same (S-trials), or different speed (D-trials). To evaluate whether the observed modulation of the S2 response depended on the exact speeds during S1 and S2, we performed a separate analysis of trials with S2 moving at the highest speed (either $10^{\circ} \%$ or $16^{\circ} / \mathrm{s}$ ). This analysis was performed on a subset of cells with sufficient numbers of S- and D-trials $(n=30)$. Among these cells, we found no neurons that reversed their preference for trial type, and $50 \%$ of cells with CEs showed a consistent preference (either $\mathrm{S}>\mathrm{D}$ or $\mathrm{D}>\mathrm{S}$ ), regardless of S2 speed. Overall, there was no significant difference in the average CEs calculated for trials with slow and fast S2 (slow S2, $0.65 \pm 0.025$; fast S2, $0.62 \pm 0.022 ; p=$ 0.65 , Wilcoxon sign-rank test), supporting the interpretation that the observed effects reflected the comparison between S1 and S2 and did not depend on their absolute speed.

During each recording session, we measured the accuracy of speed discrimination by presenting the monkeys with the range of speed differences. This allowed us to examine whether these differences affected the CEs recorded in individual neurons. We found that both types of effects decreased as speed differences decreased (Fig. 10F; S > D, blue symbols and $D>S$, red symbols). This relationship was highly significant $(p=$ $0.00006, r^{2}=0.812$, Pearson's correlation), providing further confirmation that the observed modulation of responses to S2 reflects a sensory comparison process required by our task. This hypothesis received further support when we examined whether the CE was also present during the passive fixation task. This analysis was performed for a small subset of neurons with a sufficient number of trials recorded during the active and passive tasks $(n=21)$. The result, plotted in Figure $11 A$, shows the average CE based on the combined activity of both NS and BS cells for the two cell groups $(S>D$ and $D>S$ ). This effect is also shown for individual neurons in the form of a scatterplot (Fig. $11 B)$, illustrating an overall reduction of CE during passive fixation ( $p=0.004$, Wilcoxon sign-rank test). The finding that the CEs become prominent when animals are required to compare 

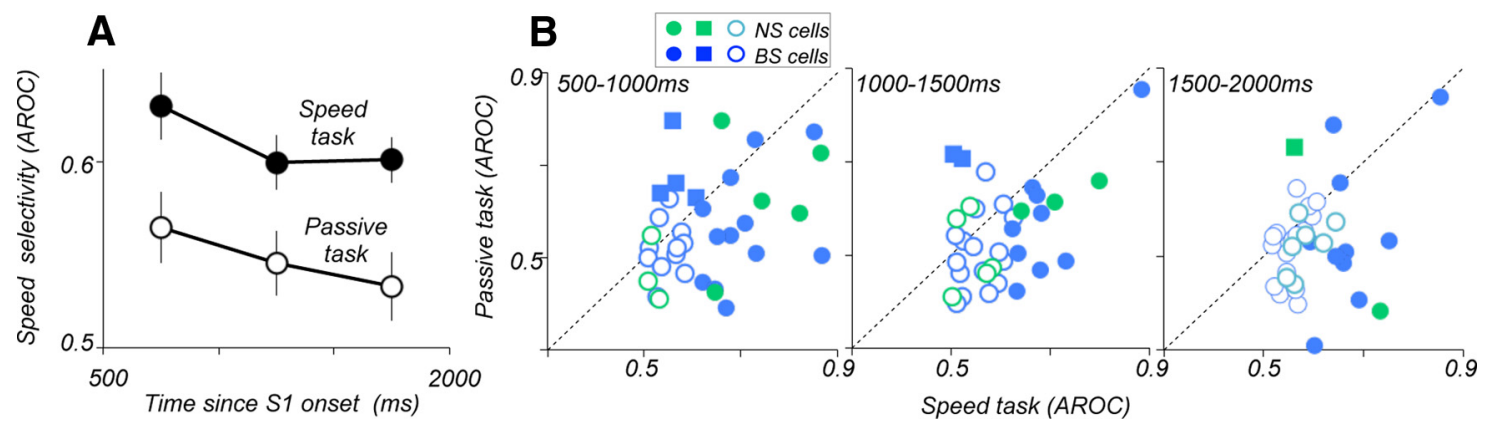

Figure 9. Speed-selective delay activity is weakened during passive fixation. $A$, Average delay selectivity during the speed and the passive fixation tasks. Each data point represents average activity of $27 \mathrm{BS}$ and $7 \mathrm{NS}$ neurons during three consecutive $500 \mathrm{~ms}$ periods of the delay. $B$, Speed selectivity of individual neurons contributing to data shown in $A$. NS and BS neurons with significant activity in the speed task $(\mathbf{)}$ ), and in the passive task alone $(\square)$ are shown separately. $\bigcirc$, neurons with no significant selectivity in either task. Selectivity was greater during the speed task throughout the delay (early: $p=0.005$; middle: $p=0.003$; late: $p=0.0001$, Wilcoxon sign-rank test).

and report speed differences provide yet another confirmation that the observed modulation of S2 responses by S1 represents an important process underlying the speed comparison task performed by our monkeys.

\section{Abstract representation of "same" and "different" trials}

The finding that S-trials and D-trials were represented by distinct groups of DLPFC neurons parallels the effects we recently reported while recording from the same set of neurons during the direction comparison task (Hussar and Pasternak, 2012). We wondered whether during the speed and the direction tasks a given neuron's trial preferences coincide and, thus, reflect a general preference for "same" or "different" trials, independent of stimulus features being compared (Fig. 12A). We addressed this question by analyzing CEs in cells with a sufficient number of trials recorded during each task $(n=70)$. The data for individual neurons, color-coded by the trial-type preference exhibited during the speed task, are plotted in Figure $12 B$. The data show that many DLPFC neurons tend to prefer the same type of trial during the two tasks. Among neurons with CEs during the speed task, $74 \%$ preferred the same trial type during the direction task. Furthermore, AROC values between tasks were strongly correlated $\left(p=3.0 \times 10^{-8}, r^{2}=0.604\right.$, Pearson's correlation), suggesting that many

DLPFC neurons reflect an abstract "same" or "different" rule, regardless of stimulus conditions or task structure.

\section{Choice-related activity}

The results above showed that, at the time of the comparison phase of the task, DLPFC neurons carry "same" and "different" signals that could be used in the monkey's perceptual report. To determine the participation of these neurons in decision-making, we examined whether their activity predicts "same" and "differ-
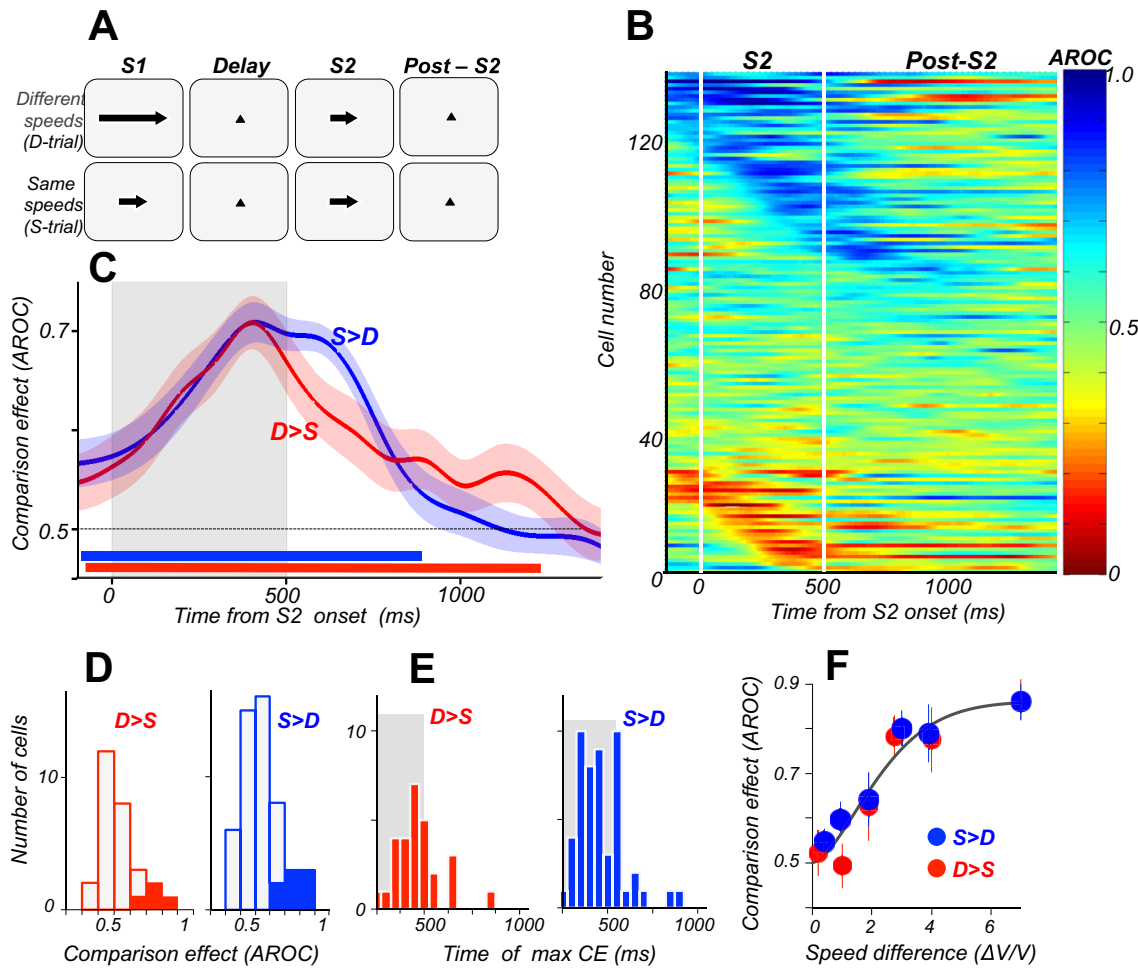

E

Figure 10. Modulation of $S 2$ responses by the preceding speed. $A$, Diagram of the two types of trials used to evaluate CEs Longer and shorter arrows indicate faster and slower speeds, respectively. $\boldsymbol{B}$, CEs in individual neurons quantified with ROC analysis $(B S=103, N S=33)$. AROC values $>0.5$ (cooler colors) indicate higher activity on $S$-trials; AROC values $<0.5$ (warmer colors) indicate stronger activity on D-trials. Neurons were sorted by timing and sign of their CES. C, Average CEs for $S>D(n=51)$ and $D>S(n=28)$ cells during $S 2$ and post-S2 periods. Shadings indicate \pm SEM. Period of significant effect is indicated by a thick horizontal line (red for $S>D$; blue for $D>S$ ). $D$, Distribution of $S>D$ and $D>S$ effects contributing to the average data shown in C. $E$, Distribution of timing of maximal CEs for $S>D$ (blue) and D $>S$ (red) neurons. Mean latencies: $S>D$ cells, $506 \pm 47 \mathrm{~ms}$; $D>S$ cells, $458 \pm 53 \mathrm{~ms}$. $\boldsymbol{F}$, Relationship between the CE ( $y$-axis) and speed difference ( $x$-axis). Each data point represents maximal CE at a given speed difference for each neuron. Both cell types showed a decreased CE with smaller differences in speed. 


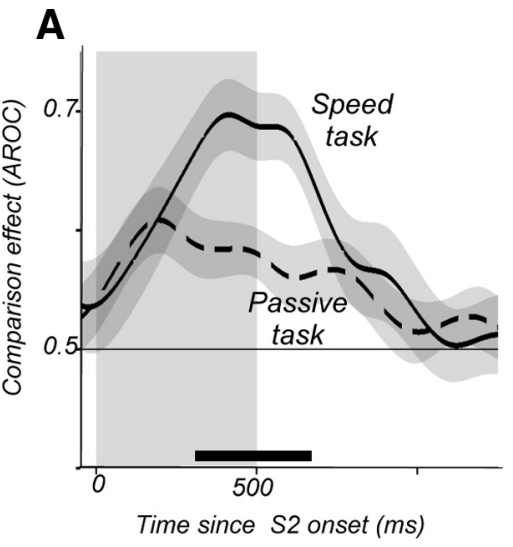

B

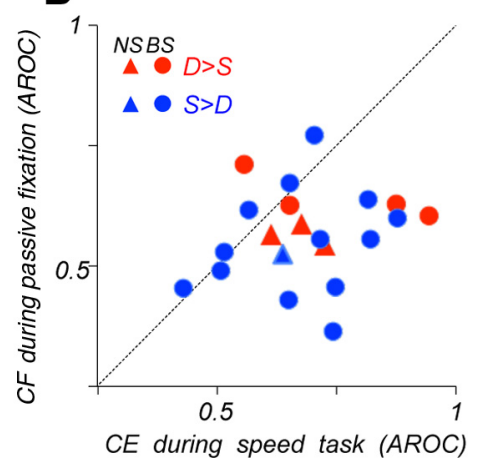

Figure 11. Attenuation of comparison effects during passive fixation. $A$, Average comparison effects during the speed and the passive fixation tasks computed for cells with sufficient number of trials during the two tasks ( $N S=4, B S=17$ ). Trial-type preference was based on S2 responses during the speed task (see Materials and Methods). $\boldsymbol{B}$, Cell-by-cell comparison effects recorded during the two tasks. Each data point represents activity recorded during $400-600 \mathrm{~ms}$ after the onset of $S 2$. The effects were weaker during the passive task (Wilcoxon sign-rank test, $p=0.004$ ).

or "different" $(N=27 ; 25 \%)$ reports. Figure $13 A$ shows the average $\mathrm{CP}$ for all neurons with higher activity before each of the two types of reports. The activity predictive of both types of reports was highly significant (500 ms window centered at time of maximal effect; "different report," $\mathrm{CP}=0.59 \pm 0.03, p=0.004$; "same report," $\mathrm{CP}=$ $0.645 \pm 0.026, p=0.0002$, Mann-Whitney U Test). Although visual inspection of average CPs for the two types of report-related activity suggests that the maximal effect for activity predictive of "different" reports occurs earlier, at the time of maximal CP this difference was not significant ("different report," $526 \mathrm{~ms} \pm 37$; “same report," 567 $\mathrm{ms} \pm 53 ; p=0.39$ ).

The CPs of both "same" and "different" reports showed a similar time course to the CEs for S $>$ D and D $>$ S cells. This similarity suggested a possible relationship between these two effects, prompting us to examine their co-occurrence in individual neurons. Figure $13 B$ shows that comparison and choice-related effects co-occurred in nearly $30 \%$ of neurons. Approximately a third of cells (27\%) showed only comparisonrelated activity, a minority (17\%) exhibited only CPs with no CE, and the remaining $27 \%$ showed no comparison- or choicerelated activity. To examine the relationship between CPs and CEs, we compared the strength and timing of the effects that co-occurred in the same neurons. Figure $14 A$ shows the average CE (solid lines) and CP (broken lines) for all neurons with both effects $(n=32)$.

The data for $\mathrm{S}>\mathrm{D}$ cells (blue curves, $n=23$ ) and $\mathrm{D}>\mathrm{S}$ cells (red curves, $n=9$ ) are plotted separately. Visual inspection of the average data suggests that CEs preceded CPs. We quantified this
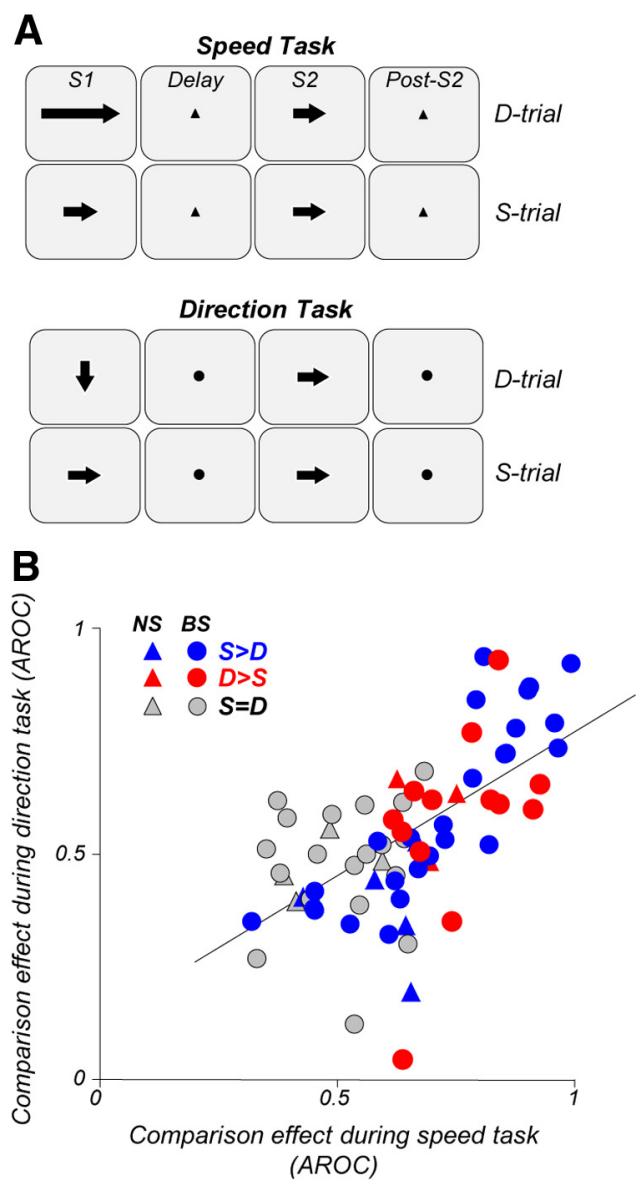

Figure 12. Representation of "Same" and "Different" effects across tasks. A, Diagram of the two types of trials used to evaluate comparison effects during the speed (top) and direction (bottom) discrimination tasks. The two tasks were run in separate blocks. $\boldsymbol{B}$, Correlation between comparison effects calculated in both tasks for all neurons $(N=70)$. Neurons are color-coded for trial-type preference exhibited during the speed discrimination task $(S>D=$ blue, $D>S=$ red, $S=D$, gray). The data show a strong correlation between trial type preference of individual neurons during the two tasks $\left(R^{2}=0.604\right.$, Pearson's correlation, $p=3.0 \times 10^{-8}$ ).

observation by comparing the times of maximal CE and CPs in individual neurons for the two types of effects. Figure $14 B$ shows that, in the majority of neurons with co-occurring two types of activity, CP trailed the CE by $\sim 200 \mathrm{~ms}$ for $\mathrm{S}>\mathrm{D}$ neurons $(p=$ 0.0078, Wilcoxon sign-rank task) and by $190 \mathrm{~ms}$ for $\mathrm{D}>\mathrm{S}$ neurons ( $p=0.023$, Wilcoxon sign-rank task). This earlier emergence of comparison-related activity is consistent with the possibility that this activity may be used in the decision process. To further explore this possibility, we asked whether the trial-type ("S-trial" and "D-trial") and report-type activity ("same" and "different") correlate with each other in individual neurons. Scatterplots in Figure 14C show a strong correlation between comparison and choice-related activity both during S2 (left plot, 300-500 ms; $p=0.00002, r^{2}=0.43$, Pearson's correlation) and during the post-S2 period leading to the perceptual report (right plot, 900-1100 ms; $p=0.000008, r^{2}=0.48$, Pearson's correlation). This correlation indicates that neurons preferring S-trials (red markers, S > D) also tended to fire more before "same" reports, whereas cells that preferred D-trials (blue, markers, D > S) also showed higher rates before "different" reports. Figure $14 D$ shows the strength of the correlation between CE and CP as a function of time, starting at the end of the delay, during S2, and post-S2 periods. The time course of this correlation highlights 
two important aspects of these results. First, during the period of late delay immediately preceding S2, there was no correlation between $\mathrm{CE}$ and $\mathrm{CP}$, despite some neurons showing CEs $>0.5$ at this time (Fig. 10). This lack of correlation supports the notion that these effects reflect primarily delay selectivity and are unlikely to contribute to the decision process. Second, the correlation between CE and CP was consistent toward the end of the trial, to the point immediately preceding the motor response. This relationship between CP and CE provides strong evidence that the information regarding the sensory comparison may be used in the decision process.

\section{Discussion}

We showed that during the speed comparison task, many DLPFC neurons, both NS putative interneurons and BS pyramidal neurons, exhibited speed-selective responses. During the delay, BS neurons were more active and exhibited reliable but brief periods of stimulus selectivity. During S2, responses of NS and BS neurons were modulated by speed differences between $\mathrm{S} 1$ and S2, the effect that weakened when these differences were small. Finally, the activity of many neurons with CEs also carried signals predictive of the animal's choice, and we found strong correlation between comparison and choice-related signals, which tended to be of the same sign.

\section{Responses to different dimensions of motion}

We found that selectivity for behaviorally relevant speeds in DLPFC was reminiscent of speed tuning observed in MT (Maunsell and Van Essen, 1983; Liu and Newsome, 2003; Nover et al., 2005). As in MT, some neurons preferred slow, some fast, and some intermediate speeds; and as in MT, their tuning was well described by a log-Gaussian function (Nover et al., 2005). We should note that the relatively narrow range of speeds used in our task did not always allow us to present the most optimal stimuli for a given neuron. This was particularly true for cells preferring the fastest speeds. Those cells were labeled as high-pass, although it is possible that the highest speeds we used were optimal and their responses were actually in a bandpass category. Thus, we were likely to underestimate the incidence of bandpass neurons. On the other hand, nearly half of our neurons exhibited lowpass tuning, most likely because of stimuli being presented at the fovea, the region likely to be represented by motion-
A

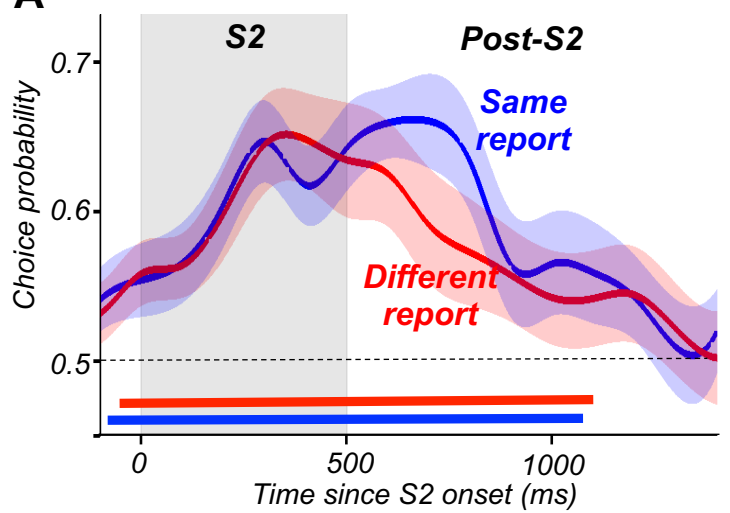

B
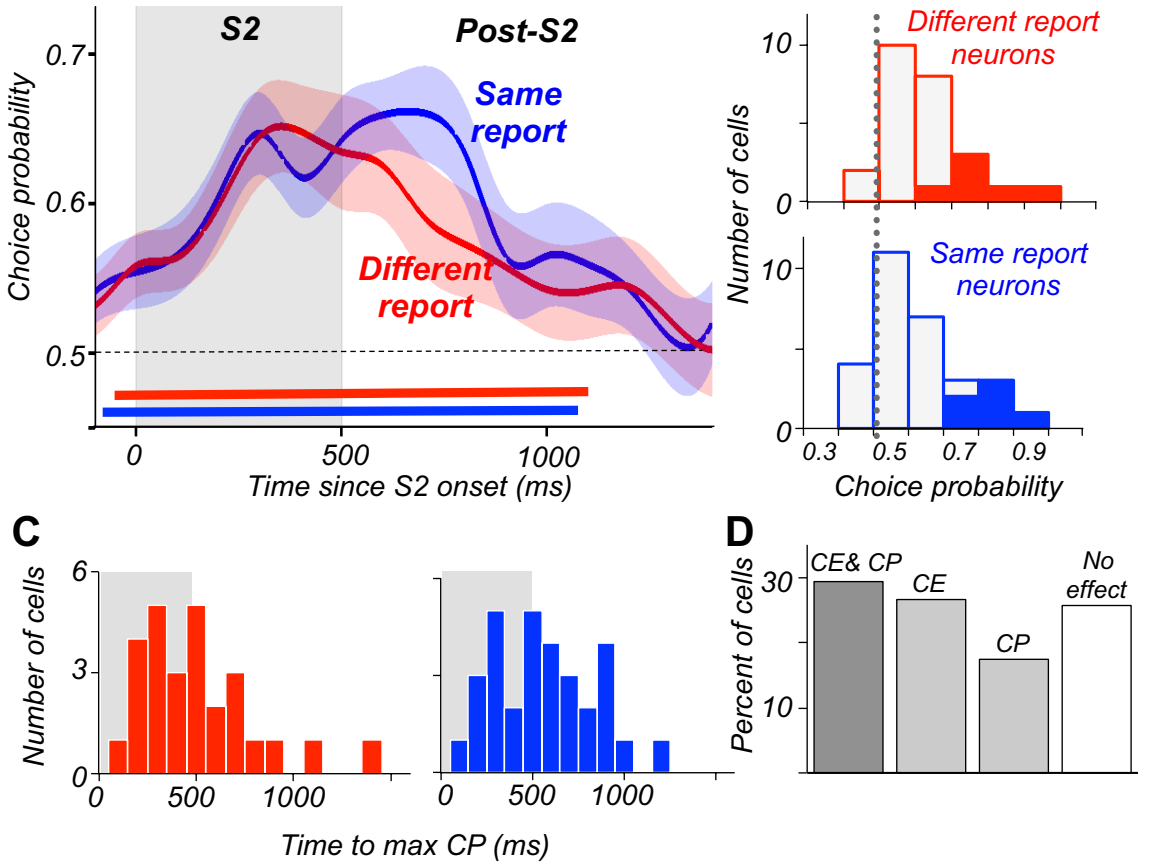

Figure 13. Activity during and after $\$ 2$ predicts upcoming perceptual report. $A, C P$ of neurons with higher activity before "different reports" ( $n=27)$ and higher activity before "same reports" $(n=31)$. Thick colored lines along the $x$-axis indicate period of significance (Mann-Whitney U test, $p<0.05$ ). Shadings represent $\pm S E M$. B, Distribution of CP values contributing to the data plotted in $\boldsymbol{A}$. (Ps were computed for the period of $300-800 \mathrm{~ms}$ after $\$ 2$ onset. C, Time of maximal CP for neurons contributing to CP curves shown in A. Average maximal CP times for "same" and "different" reports were similar ("same," $526 \mathrm{ms;}$ and "different," $567 \mathrm{~ms} ; p=0.39)$. $D$, Incidence of $C E$ and $C P$ signals co-occurring in the same neurons ( $C P$ and $C E, n=32 ; C E, n=29 ; C P, n=19$; no effect, $n=28)$.
A

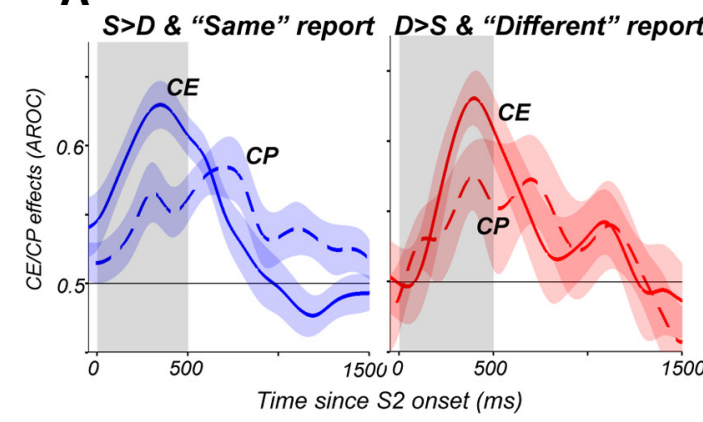

C

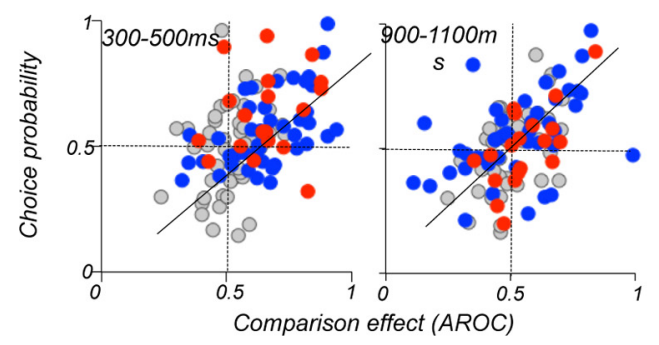

B
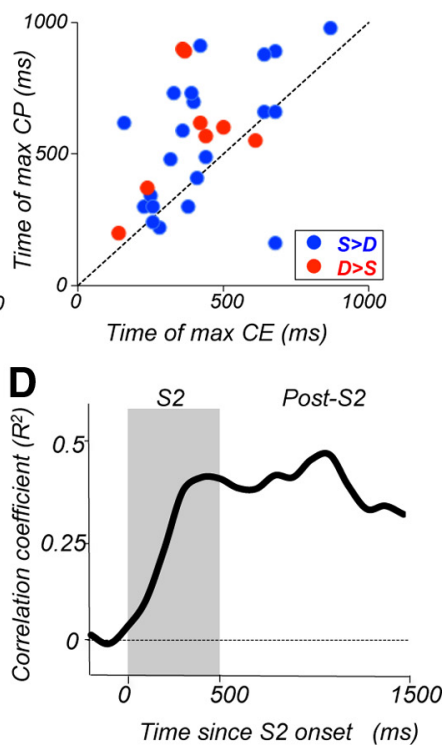

Figure 14. Relationship between comparison and choice-related activity. $\boldsymbol{A}, \mathrm{CEs}$ (solid lines) and CP (broken lines) in $\mathrm{S}>$ D neurons $(n=23)$ and $D>S$ neurons $(n=9)$. Only neurons with co-occurring CE and CP (see Fig. 13D) were used. $\boldsymbol{B}$, Times of maximal CP and CE for individual neurons. Note that the time of maximal $C P$ trailed the time of maximal CE $(p=$ 0.001 , Wilcoxon sign-rank test). $C$, Correlation between CE and CP for individual $S>D$ and D $>S$ cells at $300-500 \mathrm{~ms}(p=$ $2.0 \times 10^{-5}, R^{2}=0.43$, Pearson's correlation) and at $900-1100 \mathrm{~ms}\left(p=8.0 \times 10-6, R^{2}=0.48\right.$, Pearson's correlation) after $S 2$ onset. $D$, Coefficient of correlation between CE and CP, computed across time (late delay, during $S 2$ and post-S2). 
processing neurons preferring lower speeds (Mikami et al., 1986a; Lagae et al., 1993).

The similarity between speed representation in DLPFC and in area MT parallels the similarity between responses to motion direction in the two areas (Zaksas and Pasternak, 2006). As in MT, the majority of neurons selective for speed were also selective for direction. However, in contrast to MT, where these two properties coexist in passively fixating or anesthetized monkeys (Maunsell and Van Essen, 1983), in the DLPFC each feature revealed itself largely when it was behaviorally relevant (Hussar and Pasternak, 2009, 2012).

We recently showed that, during passive fixation, direction selectivity of both NS and BS cells is greatly decreased (Hussar and Pasternak, 2009, 2012), the finding analogous to the loss of speed selectivity during passive fixation observed here. We also found that active shifts of attention from direction to speed decreased direction selectivity primarily in NS neurons, suggesting their greater sensitivity to task demands (Hussar and Pasternak, 2009, 2012). We were not able to determine whether NS neurons would show a loss in speed selectivity during direction discrimination task because during that task we did not vary speeds. However, based on our previous observation that the two types of selectivity can be modulated independently by behavioral relevance (Hussar and Pasternak, 2009), we speculate that an active shift in attention from speed to another feature would result in weakened selectivity for speed and that this effect would be greater in NS cells.

We observed that, while both NS and BS cells were tuned for stimulus speed, the selectivity of NS neurons was more prominent, the difference consistent with responses of these neurons to other stimulus dimensions (Diester and Nieder, 2008; Hussar and Pasternak, 2009). One explanation for the higher selectivity of NS neurons could be their higher firing rates (Connors and Gutnick, 1990) and the greater dynamic range with which to encode stimulus differences. Furthermore, whereas NS cells are characterized by higher trial-to-trial variability (Fano factor), their greater stimulusdriven decrease in the Fano factor compared with BS cells (Mitchell et al., 2007) may also enhance their selectivity.

\section{Speed-selective delay activity}

We found that delay activity was characterized by transient periods of selectivity in individual neurons that was largely independent of their preferences during $\mathrm{S} 1$ and that this activity was more common in BS neurons. These features parallel our recent observations of delay activity recorded during the direction task (Hussar and Pasternak, 2012), suggesting that maintenance of the information about speed and direction may be governed by similar or common mechanisms.

Although the prominent role for putative pyramidal neurons in maintaining sensory information is in line with predictions of some computational models (Wang, 2001; Brody et al., 2003), the transient nature of their delay activity recorded during our task is not. Indeed, many models assume that in individual neurons this activity is persistent (Durstewitz et al., 2000; Wang, 2001; Brody et al., 2003; Engel and Wang, 2011). These models, initially developed to account for the sustained delay activity commonly observed during spatial memory tasks, were eventually extended to other stimulus features (Brody et al., 2003; Engel and Wang, 2011). The absence of persistent delay activity in individual neurons does not necessarily rule out the utility of transient activity appearing at different times in different neurons. Indeed, when averaged across neurons, stimulus selective delay activity, decreased when these features were not relevant to the task (Fig. 9)
(Hussar and Pasternak, 2012). The possibility that stimulus information is distributed among neurons and can be maintained throughout the delay is consistent with population averages of delay activity for other stimulus features (Miller et al., 1996; Romo and Salinas, 2003; Shafi et al., 2007; Jun et al., 2010; Cromer et al., 2011).

\section{Memory-guided comparison effects}

During S2, responses of many neurons reflected the difference between the two comparison speeds. Although in a small subset of cells we could not rule out stimulus selectivity "bleeding in" from the late delay, contributing to CEs (Engel and Wang, 2011), the majority of these effects occurred in the absence of any delay selectivity. This raises the question of how information about the preceding speed becomes available during S2. One possibility is that changes in synaptic facilitation induced by $\mathrm{S} 1$ alter responses during S2, effectively storing the preceding speed at the level of the synapse (Mongillo et al., 2008). It is also possible that comparison signals in the DLPFC are the result of its interactions with the upstream area MT. In this scenario, putative pyramidal cells carrying speed-selective delay activity could be supplying MT with the information about the preceding speed, ensuring that MT has access not only to the current but also to the previous stimulus. Indeed, we recently reported comparison effects in MT analogous to those observed in DLPFC, with three distinct groups of cells representing either S-trials or D-trials (Lui and Pasternak, 2011). Based on the nature and the time course of these effects, we hypothesized that the circuitry underlying comparisons of motion direction include both regions and that the early comparison effect observed in MT may trigger that process (Hussar and Pasternak, 2012). An analogous mechanism may be involved in speed comparisons. Future studies involving local inactivation of either MT or the DLPFC will provide insights into their respective contributions to the underlying process.

We observed a striking consistency in the sign of comparison effects recorded during the speed or direction discrimination tasks (Fig. 12), with many neurons preferring the same trial type during both tasks. This consistency suggests that DLPFC neurons represent selectivity for "same/different" abstract rule governing the task, independent of stimulus dimension. Similar rule encoding in prefrontal cortex has been observed by others (Wallis et al., 2001; Genovesio et al., 2005; Bongard and Nieder, 2010). We speculate that the presence of "same/different" rule is not an inherent property of DLPFC. Rather, it may have emerged while monkeys were learning "same/different" comparison tasks, attesting to the documented capability of prefrontal neurons to acquire abstract rules (Sakai, 2008).

\section{Choice-related activity and CEs}

Many studies examining choice-related activity used tasks requiring identification of specific stimulus features, such as motion direction or depth (Nienborg et al., 2012). These studies showed that the neurons carrying the most reliable signals are more likely to contribute to the decision process. In our task, the observed comparison effects may be analogous to direction selectivity recorded during tasks requiring animals to report motion direction, supporting the idea that perceptual decisions are based on neurons carrying the most reliable information needed for those decisions.

The co-occurrence of comparison effects and choice-related activity in the same neurons and the strong correlation between them resembles the relationship between the two types of effects recorded during comparisons of motion direction (Hussar and 
Pasternak, 2012), pointing to a generalized perceptual decision mechanism that uses the results of sensory comparisons to transform them into behavioral reports.

In conclusion, our work demonstrates striking similarities between representations in DLPFC of speed and direction during comparison tasks. During S1 and S2, response selectivity for these two fundamental dimensions of motion resembles stimulusselective responses of MT neurons, a likely source of motion signals reaching the DLPFC. In both direction and speed tasks, BS neurons were more active during the delay and were more likely to carry anticipatory and stimulus-selective activity. The similarity between the two dimensions also extended to the transient nature of stimulus-selective delay activity, to the way DLPFC neurons represented comparison signals and to their strong correlation with decision-related activity. The parallels in activity associated with two distinct sensory dimensions suggests the operation of common rules governing the way stimuli are represented and used during memory-guided sensory comparisons. Finally, our results highlight the precision and flexibility with which DLPFC represents fundamental stimulus dimensions, suggesting that during sensory comparisons it is likely to provide the upstream motion-processing neurons with highly specific information about both visual motion and its behavioral context.

\section{References}

Barbas H (1988) Anatomic organization of basoventral and mediodorsal visual recipient prefrontal regions in the rhesus monkey. J Comp Neurol 276:313-342. CrossRef Medline

Bongard S, Nieder A (2010) Basic mathematical rules are encoded by primate prefrontal cortex neurons. Proc Natl Acad Sci U S A 107:2277-2282. CrossRef Medline

Britten KH, Newsome WT, Shadlen MN, Celebrini S, Movshon JA (1996) A relationship between behavioral choice and the visual responses of neurons in macaque MT. Vis Neurosci 13:87-100. CrossRef Medline

Brody CD, Romo R, Kepecs A (2003) Basic mechanisms for graded persistent activity: discrete attractors, continuous attractors, and dynamic representations. Curr Opin Neurobiol 13:204-211. CrossRef Medline

Connors BW, Gutnick MJ (1990) Intrinsic firing patterns of diverse neocortical neurons. Trends Neurosci 13:99-104. CrossRef Medline

Contreras D, Palmer L (2003) Response to contrast of electrophysiologically defined cell classes in primary visual cortex. J Neurosci 23:6936-6945. Medline

Cromer JA, Roy JE, Buschman TJ, Miller EK (2011) Comparison of primate prefrontal and premotor cortex neuronal activity during visual categorization. J Cogn Neurosci 23:3355-3365. CrossRef Medline

Diester I, Nieder A (2008) Complementary contributions of prefrontal neuron classes in abstract numerical categorization. J Neurosci 28: 7737-7747. CrossRef Medline

Durstewitz D, Seamans JK, Sejnowski TJ (2000) Neurocomputational models of working memory. Nat Neurosci 3:1184-1191. CrossRef Medline

Efron B, Tibshirani RJ (1993) An introduction to the bootstrap. New York: Chapman and Hall.

Engel TA, Wang XJ (2011) Same or different? A neural circuit mechanism of similarity based pattern-match decision making. J Neurosci 31:6982-6996.

Genovesio A, Brasted PJ, Mitz AR, Wise SP (2005) Prefrontal cortex activity related to abstract response strategies. Neuron 47:307-320. CrossRef Medline

Gold JI, Shadlen MN (2007) The neural basis of decision making. Annu Rev Neurosci 30:535-574. CrossRef Medline

Hartigan JA, Hartigan PM (1985) The dip test of unimodalit. Ann Statist 13:70-84. CrossRef

Hussar C, Pasternak T (2010) Trial-to-trial variability of the prefrontal neurons reveals the nature of their engagement in a motion discrimination task. Proc Natl Acad Sci U S A 107:21842-21847. CrossRef Medline

Hussar CR, Pasternak T (2009) Flexibility of sensory representations in prefrontal cortex depends on cell type. Neuron 64:730-743. CrossRef Medline

Hussar CR, Pasternak T (2012) Memory-guided sensory comparisons in the prefrontal cortex: contribution of putative pyramidal cells and interneurons. J Neurosci 32:2747-2761. CrossRef Medline

Johnston K, DeSouza JF, Everling S (2009) Monkey prefrontal cortical py- ramidal and putative interneurons exhibit differential patterns of activity between prosaccade and antisaccade tasks. J Neurosci 29:5516-5524. CrossRef Medline

Jun JK, Miller P, Hernández A, Zainos A, Lemus L, Brody CD, Romo R (2010) Heterogeneous population coding of a short-term memory and decision task. J Neurosci 30:916-929. CrossRef Medline

Lagae L, Raiguel S, Orban GA (1993) Speed and direction selectivity of macaque middle temporal neurons. J Neurophysiol 69:19-39. Medline

Liu J, Newsome WT (2003) Functional organization of speed tuned neurons in visual area MT. J Neurophysiol 89:246-256. Medline

Liu J, Newsome WT (2005) Correlation between speed perception and neural activity in the middle temporal visual area. J Neurosci 25:711-722. CrossRef Medline

Lui LL, Pasternak T (2011) Representation of comparison signals in cortical area MT during a delayed direction discrimination task. J Neurophysiol 106:1260-1273. CrossRef Medline

Maunsell JH, Van Essen DC (1983) Functional properties of neurons in middle temporal visual area of the macaque monkey. I. Selectivity for stimulus direction, speed, and orientation. J Neurophysiol 49:1127-1147. Medline

McCormick DA, Connors BW, Lighthall JW, Prince DA (1985) Comparative electrophysiology of pyramidal and sparsely spiny stellate neurons of the neocortex. J Neurophysiol 54:782-806. Medline

Mikami A, Newsome WT, Wurtz RH (1986a) Motion selectivity in macaque visual cortex. II. Spatiotemporal range of directional interactions in MT and V1. J Neurophysiol 55:1328-1339. Medline

Mikami A, Newsome WT, Wurtz RH (1986b) Motion selectivity in macaque visual cortex. I. Mechanisms of direction and speed selectivity in extrastriate area MT. J Neurophysiol 55:1308-1327. Medline

Miller EK, Cohen JD (2001) An integrative theory of prefrontal cortex function. Annu Rev Neurosci 24:167-202. CrossRef Medline

Miller EK, Erickson CA, Desimone R (1996) Neural mechanisms of visual working memory in prefrontal cortex of the macaque. J Neurosci 16: 5154-5167. Medline

Mitchell JF, Sundberg KA, Reynolds JH (2007) Differential attentiondependent response modulation across cell classes in macaque visual area V4. Neuron 55:131-141. CrossRef Medline

Mongillo G, Barak O, Tsodyks M (2008) Synaptic theory of working memory. Science 319:1543-1546. CrossRef Medline

Nienborg H, Cohen MR, Cumming BG (2012) Decision-related activity in sensory neurons: correlations among neurons and with behavior. Annu Rev Neurosci 35:463-483. CrossRef Medline

Nover H, Anderson CH, DeAngelis GC (2005) A logarithmic, scaleinvariant representation of speed in macaque middle temporal area accounts for speed discrimination performance. J Neurosci 25:1004910060. CrossRef Medline

Pasternak T (1987) Discrimination of differences in speed and flicker rate depends on directionally selective mechanisms. Vis Res 27:1881-1890. CrossRef Medline

Petrides M, Pandya DN (2006) Efferent association pathways originating in the caudal prefrontal cortex in the macaque monkey. J Comp Neurol 498:227-251. CrossRef Medline

Rodman HR, Albright TD (1987) Coding of visual stimulus velocity in area MT of the macaque. Vis Res 27:2035-2048. CrossRef Medline

Romo R, Salinas E (2003) Flutter discrimination: neural codes, perception, memory and decision making. Nat Rev Neurosci 4:203-218. CrossRef Medline

Sakai K (2008) Task set and prefrontal cortex. Annu Rev Neurosci 31:219245. CrossRef Medline

Schall JD, Morel A, King DJ, Bullier J (1995) Topography of visual cortex connections with frontal eye field in macaque: convergence and segregation of processing streams. J Neurosci 15:4464-4487. Medline

Shafi M, Zhou Y, Quintana J, Chow C, Fuster J, Bodner M (2007) Variability in neuronal activity in primate cortex during working memory tasks. Neuroscience 146:1082-1108. CrossRef Medline

Wallis JD, Anderson KC, Miller EK (2001) Single neurons in prefrontal cortex encode abstract rules. Nature 411:953-956. CrossRef Medline

Wang XJ (2001) Synaptic reverberation underlying mnemonic persistent activity. Trends Neurosci 24:455-463. CrossRef Medline

Zaksas D, Pasternak T (2006) Directional signals in the prefrontal cortex and in area MT during a working memory for visual motion task. J Neurosci 26:11726-11742. CrossRef Medline 